\title{
Réviser les tarifs de cubage pour prendre en compte l'évolution de la ressource au Cameroun
}

Gauthier LIGOT ${ }^{2}$

Nicolas DUBART ${ }^{1}$

Mauriad TCHOWO HAPI

Sébastien BAUWENS ${ }^{2}$

Jean-Louis DouCET ${ }^{2}$

Adeline FAYOLLE ${ }^{2}$

${ }^{1}$ Nature+ asbl

Winstar Park

Rue Provinciale 62

1301 Wavre

Belgique

2 Université de Liège

Gembloux Agro-Bio Tech

TERRA Teaching and Research Center

Forest is life

Passage des Déportés 2

5030 Gembloux

Belgique

Auteur correspondant /

Corresponding author:

Gauthier Ligot - gligot@uliege.be

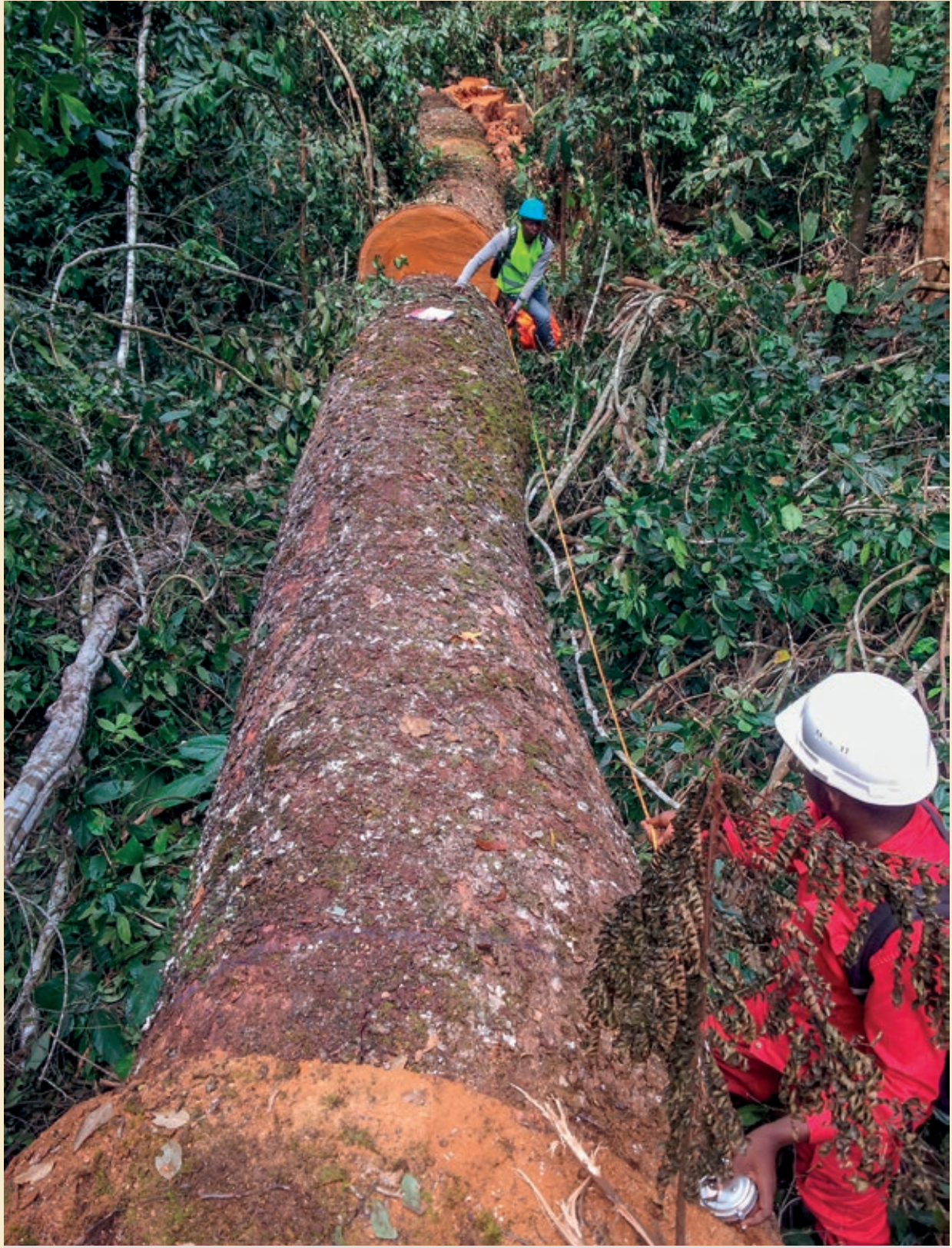

Photo 1.

Estimation du volume par la méthode des billons successifs, mesure de la longueur de la bille. Photo N. Dubart.

Doi : 10.19182/bft2018.338.a31677 - Droit d'auteur @ 2018 , Bois et Forêts des Tropiques @ Cirad - Date de soumission : 7 décembre 2017 ; date d'acceptation : 11 juillet 2018 ; date de publication : $1^{\text {er }}$ décembre 2018.

\section{Ocirad (6)}

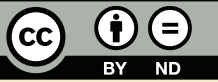

Licence Creative Commons :

Attribution - Pas de Modification 4.0 International. Attribution-NoDerivatives 4.0 International (CC BY-ND 4.0)
Citer l'article / To cite the article

Ligot G., Dubart N., Tchowo Hapi M., Bauwens S., Doucet J.-L., Fayolle A., 2018. Réviser les tarifs de cubage pour prendre en compte l'évolution de la ressource au Cameroun. Bois et Forêts des Tropiques, $n^{\circ} 338$ : 57-71. Doi : https://doi.org/10.19182/bft2018.338.a31677 


\section{RÉSUMÉ}

\author{
Réviser les tarifs de cubage \\ pour prendre en compte l'évolution \\ de la ressource au Cameroun
}

La connaissance du volume exploitable est une information essentielle tant pour la gestion que pour le contrôle de l'exploitation forestière. En Afrique centrale, l'estimation des volumes repose essentiellement sur l'utilisation de tarifs de cubage à une entrée, spécifique à chaque essence, et prédisant le volume exploitable à partir du diamètre de l'arbre. Or, récemment, de nombreux acteurs de la gestion forestière au Cameroun rapportent une inadéquation entre les volumes commerciaux estimés avec les tarifs de cubage imposés par l'administration et les volumes estimés à partir des mesures de la longueur et du diamètre des billes exploitées. Afin de vérifier la justesse des tarifs de cubage imposés par l'administration camerounaise, nous avons réalisé un échantillonnage destructif pour 12 essences jouant un rôle crucial dans le commerce du bois au Cameroun, et développé de nouveaux tarifs de cubage, qui ont été comparés avec les tarifs imposés par l'administration camerounaise et 52 autres tarifs de cubage disponibles dans la littérature. Dans quatre concessions forestières du Cameroun, représentatives des différentes conditions écologiques prévalant dans ce pays, 732 arbres ont été abattus et leur volume a été mesuré par la méthode des billons successifs. Des tarifs de cubage à une entrée, fonction uniquement du diamètre de référence, ont ensuite été ajustés par la méthode des moindres carrés généralisés. Notre étude confirme l'existence de biais entre les volumes mesurés et les volumes estimés en utilisant les tarifs de cubage imposés par l'administration camerounaise. En conséquence, de nouveaux tarifs de cubage et un abaque de correction sont proposés. Enfin, la majorité des tarifs de cubage testés présentaient un biais similaire qui résulte vraisemblablement d'une évolution de la ressource et des pratiques d'exploitation.

Mots-clés : aménagement forestier, allométrie, modélisation du volume, bille exploitable, essence commerciale, Cameroun, Afrique centrale.

\section{ABSTRACT}

\section{Revising volume tables to adapt to changes in timber resources in Cameroon}

Knowledge of extractable timber volumes is essential to both forestry management and logging inspections. In central Africa, estimations of timber volumes mainly rely on single-entry volume tables that are specific to each species and predict extractable volumes based on tree diameters. Recently, however, many forestry managers have been reporting inconsistencies between commercial volumes estimated by applying the volume tables imposed by the government and the volumes calculated from measurements of the length and diameter of felled logs. In order to check the accuracy of the volume tables imposed by the Cameroon government, we took destructive samples of 12 key species for Cameroon's timber trade to develop new volume tables. These were compared with those imposed by the Cameroon government and with 52 other volume tables available from the literature. In four logging concessions, chosen as representative of the different ecological conditions in Cameroon, 732 trees were felled and their volumes estimated by taking measurements of successive bole segments. The single-entry volume tables, calculated as a function of diameter only, were then fitted by the generalised least squares method. Our study confirms that there are inconsistencies between the volumes measured and the volumes estimated with the volume tables imposed by the Cameroon government. Accordingly, our study proposes new volume tables and a correction chart. Finally, the inconsistencies in the majority of the volume tables tested all produced a similar bias, which is probably due to changes in the resource and in logging practice.

Keywords: forestry management, allometry, volume modelling, extractable log, commercial species, Cameroon, central Africa.

\section{RESUMEN}

\author{
Revisión de las tarifas de cubicación \\ para adaptarlas a la evolución \\ de los recursos en Camerún
}

El conocimiento del volumen de madera explotable es una información esencial tanto para la gestión como para el control de la explotación forestal. En África central la estimación de los volúmenes reposa esencialmente en la utilización de tarifas de cubicación en una entrada, específica para cada especie, y que predice el volumen explotable a partir del diámetro del árbol. Sin embargo, numerosos actores de la gestión forestal del Camerún han informado recientemente que los volúmenes comerciales estimados con las tarifas de cubicación impuestas por la administración no corresponden a los volúmenes estimados a partir de las medidas de la longitud y del diámetro de las trozas explotadas. Para verificar la precisión de las tarifas de cubicación impuestas por la administración camerunesa, hemos realizado un muestreo destructivo para 12 especies que juegan un rol crucial en el comercio de madera en el Camerún, y hemos desarrollado nuevas tarifas de cubicación, que fueron comparadas con las tarifas impuestas por la administración camerunesa y con otras 52 tarifas de cubicación disponibles en la literatura. En cuatro concesiones forestales del Camerún, representativas de las diferentes condiciones ecológicas que prevalecen en este país, se abatieron 732 árboles y se midió su volumen mediante el método de los caballones sucesivos. Las tarifas de cubicación en una entrada, función únicamente del diámetro de referencia, se ajustaron a continuación mediante el método de los mínimos cuadrados generalizados. Nuestro estudio confirma la existencia de desviaciones entre los volúmenes medidos y los volúmenes estimados utilizando las tarifas de cubicación impuestas por la administración camerunesa. En consecuencia, se proponen nuevas tarifas de cubicación y un ábaco de corrección. En realidad, la mayor parte de las tarifas de cubicación probadas presentaban una desviación similar probablemente como resultado de una evolución de los recursos y de las prácticas de explotación.

Palabras clave: ordenación forestal, alometría, modelización del volumen, troza utilizable, especie comercial, Camerún, África central. 
En Afrique centrale, les forêts occupent près de 180 millions d'hectares, et fournissent de nombreux biens et services à un grand nombre d'acteurs. Environ $26 \%$ de ces surfaces sont allouées à des sociétés forestières pour l'exploitation du bois (De Wasseige et al., 2009). L'exploitation y est extrêmement sélective, quelques essences seulement sont ciblées, et les prélèvements sont relativement faibles en comparaison avec d'autres régions tropicales. Généralement moins d'un arbre est prélevé par hectare à chaque rotation, tous les 20 à 30 ans (Pérez et al., 2005). Les forêts de production d'Afrique centrale sont considérées comme un « modèle » de gestion (Nasi et al., 2012) bien que la durabilité des méthodes d'aménagement retenues soit questionnée sur le long terme, au-delà de deux ou trois rotations (Karsenty et Gourlet-Fleury, 2006).

La connaissance du volume exploitable est une information essentielle pour le gestionnaire forestier, et ce à différentes étapes clés de l'aménagement forestier et de la gestion forestière (Louppe et Mille, 2015). Lors de l'aménagement d'une concession forestière et grâce aux inventaires d'aménagement, la connaissance de la répartition spatiale du volume exploitable permet la subdivision de la concession en blocs quinquennaux " équivolumes », et l'établissement des budgets annuels de fonctionnement ainsi que la planification des opérations d'exploitation décrits de façon officielle dans le Plan annuel d'opération (PAO). Elle est en outre indispensable à l'élaboration du plan d'industrialisation de la société et joue un rôle déterminant dans sa rentabilité économique, un des trois piliers de la gestion durable (social, environnemental et économique).

Pour calculer le volume des arbres à partir des données de diamètre des inventaires, des équations allométriques de volume, plus généralement appelées tarifs de cubage, sont utilisées. Les tarifs de cubage sont établis pour des essences spécifiques ou des groupes d'essences, pour une zone géographique et pour une gamme de diamètres donnée (Henry et al., 2011) pour une compilation des équations disponibles pour l'Afrique subsaharienne). Si les règles d'aménagement sont relativement comparables entre les pays du bassin du Congo (Fargeot et al., 2004), les normes sont cependant spécifiques à chaque pays. Au Cameroun, les sociétés forestières ont, en outre, l'obligation légale d'utiliser les tarifs de cubage de l'administration qui sont implémentés dans le logiciel de Traitement informatique appliqué à la modélisation des aménagements (TIAMA). Or, de nombreuses sociétés rapportent une inadéquation entre les volumes prédits, calculés lors de la planification de l'exploitation, et les volumes estimés après l'exploitation à partir des mesures prises sur les parcs d'exploitation (Tchatat et al., 2008). Les volumes estimés semblent systématiquement sous-estimer les volumes réellement exploités tels qu'ils sont estimés à partir des mesures prises sur les parcs d'exploitation (cubage global). Ceci a notamment été démontré dans une étude préliminaire, pour l'assamela, le tali, et le sapelli (Fayolle et al., 2013). Par conséquent, la plupart des sociétés forestières n'exploitent qu'une partie des arbres prévus par leur PAO puisque le volume exploitable autorisé est atteint avec un nombre de tiges inférieur à celui prévu.

Il existe dès lors, tant pour les acteurs privés que publics, une réelle nécessité de revoir les tarifs de cubage pour les essences exploitées (Tchatat et al., 2008). C'est précisément dans ce contexte que s'inscrit le Projet d'amélioration continue des plans d'aménagement au Cameroun (projet ACPAC), mis en œuvre par l'association Nature ${ }^{+}$et financé par la Commission des forêts d'Afrique centrale (COMIFAC) dans le cadre du Programme de promotion de l'exploitation certifiée des forêts (PPECF). Cette étude a donc les objectifs suivants : établir de nouveaux tarifs de cubage pour les principales essences forestières exploitées au Cameroun, par la méthode destructive des billons successifs ; comparer les prédictions des tarifs de cubage ajustés lors de cette étude avec les prédictions des tarifs de cubage existants et disponibles sur la plateforme GlobAllomTree ${ }^{1}$ (Henry et al., 2013) afin d'évaluer la justesse et la précision des nouveaux tarifs de cubage par rapport à ceux disponibles ; comparer plus particulièrement les prédictions des nouveaux tarifs avec celles des tarifs de l'administration camerounaise afin de tester la présence d'un éventuel biais lié à l'utilisation de ces derniers ; enfin, vérifier l'absence de biais lors du cubage post-exploitation tel qu'il est couramment réalisé sur les parcs par les exploitants forestiers (cubage global). La comparaison entre ces différentes méthodes de cubage a notamment pour objectif de mieux comprendre l'origine des éventuels biais.

\section{Méthodes}

\section{Sites d'étude}

La collecte des données a été réalisée au Cameroun, dans quatre sites (figure 1) exploités par trois sociétés forestières différentes. Le site de Ma'an (société Wijma) est situé au sud du Cameroun près de la frontière avec la Guinée équatoriale, et présente un climat de type $\mathrm{Am}^{2}$ dans la classification de Koppen et une forêt de terre ferme de basse et moyenne altitude de type sempervirent. Les sites de Djoum (groupe Rougier) et de Mindourou (Pallisco) se situent respectivement au sud et à l'est de la Réserve de faune du Dja, et présentent une forêt de transition entre les types sempervirent et semi-caducifolié. Le climat est de type Am pour Djoum et à cheval entre les types $\mathrm{Am}_{\mathrm{m}}$ et $\mathrm{Af}^{3}$ pour Mindourou. Le site de Mbang (groupe Rougier), situé plus au nord de la Réserve de faune du Dja, présente un climat de type Af

1 http://www.globallometree.org/

2 Type de climat Am, climat tropical de mousson : température moyenne de chaque mois de l'année $>18^{\circ} \mathrm{C}$; pas de saison hivernale ; fortes précipitations annuelles (supérieures à l'évaporation annuelle); précipitations du mois le plus sec < $60 \mathrm{~mm}$ et > [100 - (précipitations annuelles moyennes) / 25].

3 Type de climat Af, climat tropical humide : précipitations tous les mois de l'année ; climat de la forêt tropicale, précipitations du mois le plus sec > $60 \mathrm{~mm}$. 


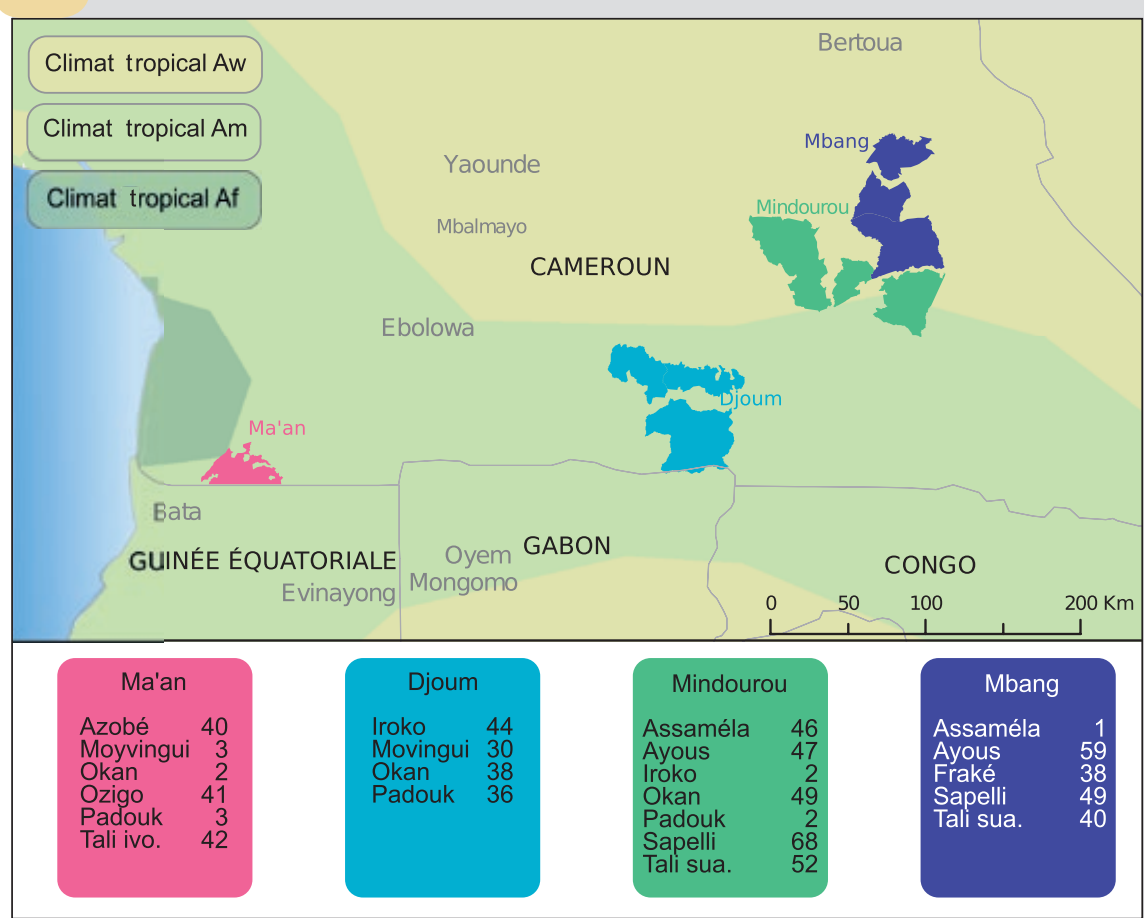

Figure 1.

Localisation des sites d'étude à Ma'an (rose), Djoum (bleu clair), Mindourou (vert) et Mbang (indigo) dans le sud du Cameroun, avec le nombre d'essences mesurées par site, positionnés dans les différents types de climat tropical de la classification de Köppen (Aw : savane ; Am : mousson ; Af : équatorial). et une forêt de type semi-caducifolié. Les données publiées par Fayolle et al. (2013) pour trois essences ayant été collectées selon la même méthodologie, et sur un des sites d'étude, le site de Mindourou, ont été incluses dans l'analyse. Les quatre sites couvrent un vaste gradient climatique avec des précipitations allant de 1500 à $2000 \mathrm{~mm}$ par an, entre respectivement les sites de Mbang et de Ma'an.

\section{Échantillonnage}

Pour établir les tarifs de cubage, douze essences ont été sélectionnées selon trois critères (tableau I), à savoir : l'intérêt commercial des essences pour les trois sociétés forestières ; la présence de tiges à collecter sur une large gamme de diamètres ; la disponibilité des tiges à sélectionner dans les zones en cours d'exploitation. Les essences retenues figurent parmi les principales essences commerciales exploitées au Cameroun pour le bois d'œuvre (De Wasseige et al., 2009, 2012). En 2010, ces essences représentaient plus de $75 \%$ des exportations de grumes et de sciage du Cameroun (ATIBT, 2012). Dans la mesure du possible, une même essence a été échantillonnée dans plusieurs sites (tableau I).

\section{Tableau I.}

Liste des essences étudiées et caractéristiques de l'échantillonnage : le nombre d'arbres échantillonnés (n) totaux (n tot.) et, pour chaque site, les moyennes (moy.), minimums (min.) et maximums (max.) des diamètres (en $\mathrm{cm}$ ) et volumes (en m³) mesurés par la méthode des billons successifs.

\begin{tabular}{|c|c|c|c|c|c|c|c|c|c|c|c|c|}
\hline \multirow[b]{2}{*}{ Nom local } & \multirow[b]{2}{*}{ Nom latin } & \multirow{2}{*}{$\begin{array}{c}n \\
\text { tot. }\end{array}$} & \multirow[b]{2}{*}{ Ma'an } & \multirow[b]{2}{*}{ Djoum } & \multirow[b]{2}{*}{ Mind. } & \multirow[b]{2}{*}{ Mbang } & \multicolumn{3}{|c|}{ Diamètre $(\mathrm{cm})$} & \multicolumn{3}{|c|}{ Volume $\left(\mathrm{m}^{3}\right)$} \\
\hline & & & & & & & moy. & $\min$. & $\max$. & moy. & $\min$. & $\max$. \\
\hline Assamela & Pericopsis elata (Harms) Meeuwen & 47 & & & 46 & 1 & 75,7 & 22,3 & 121,4 & 6,4 & 0,4 & 15,9 \\
\hline Ayous & Triplochiton scleroxylon K. Schum. & 106 & & & 47 & 59 & 105,5 & 27,1 & 172,1 & 18,1 & 0,5 & 43,0 \\
\hline Azobe & Lophira alata Banks ex C. F. Gaertn. & 40 & 40 & & & & 109,0 & 53,1 & 187,5 & 13,1 & 3,3 & 29,3 \\
\hline Fraké & Terminalia superba Engl. \& Diels & 38 & & & & 38 & 81,7 & 44,3 & 132,1 & 10,5 & 2,6 & 26,5 \\
\hline Iroko & Milicia excelsa (Welw.) C.C. Berg & 46 & & 44 & 2 & & 109,4 & 49,9 & 186,7 & 16,3 & 2,4 & 42,7 \\
\hline Movingui & Distemonanthus benthamianus Baill. & 33 & 3 & 30 & & & 102,2 & 65,0 & 137,8 & 12,0 & 3,3 & 21,9 \\
\hline Okan & Cylicodiscus gabunensis Harms & 89 & 2 & 38 & 49 & & 110,1 & 31,1 & 174,7 & 14,9 & 0,9 & 40,6 \\
\hline Ozigo & Dacryodes buettneri (Engl.) H.J. Lam & 41 & 41 & & & & 72,3 & 29,2 & 120,3 & 5,2 & 0,4 & 12,5 \\
\hline Padouk & Pterocarpus soyauxii Taub. & 41 & 3 & 36 & 2 & & 89,7 & 47,2 & 133,9 & 10,7 & 2,3 & 25,2 \\
\hline Sapelli & $\begin{array}{l}\text { Entandrophragma cylindricum } \\
\text { (Sprague) Sprague }\end{array}$ & 117 & & & 68 & 49 & 96,2 & 31,0 & 192,5 & 13,2 & 0,8 & 47,5 \\
\hline Tali ivo $^{1}$ & Erythrophleum ivorense A. Chev. & 42 & 42 & & & & 99,8 & 46,8 & 145,7 & 8,4 & 1,2 & 20,1 \\
\hline Tali sua ${ }^{1}$ & $\begin{array}{l}\text { Erythrophleum suaveolens (Guill. } \\
\text { \& Perr.) Brenan }\end{array}$ & 92 & & & 52 & 40 & 84,5 & 47,0 & 137,5 & 7,6 & 1,2 & 27,0 \\
\hline
\end{tabular}

${ }^{1}$ Tali sua et tali ivo correspondent respectivement à Eryrthrophleum suaveolens et E. ivorense (Duminil et al., 2010) et au tali Yaoundé et tali de la nomenclature du logiciel TIAMA. 


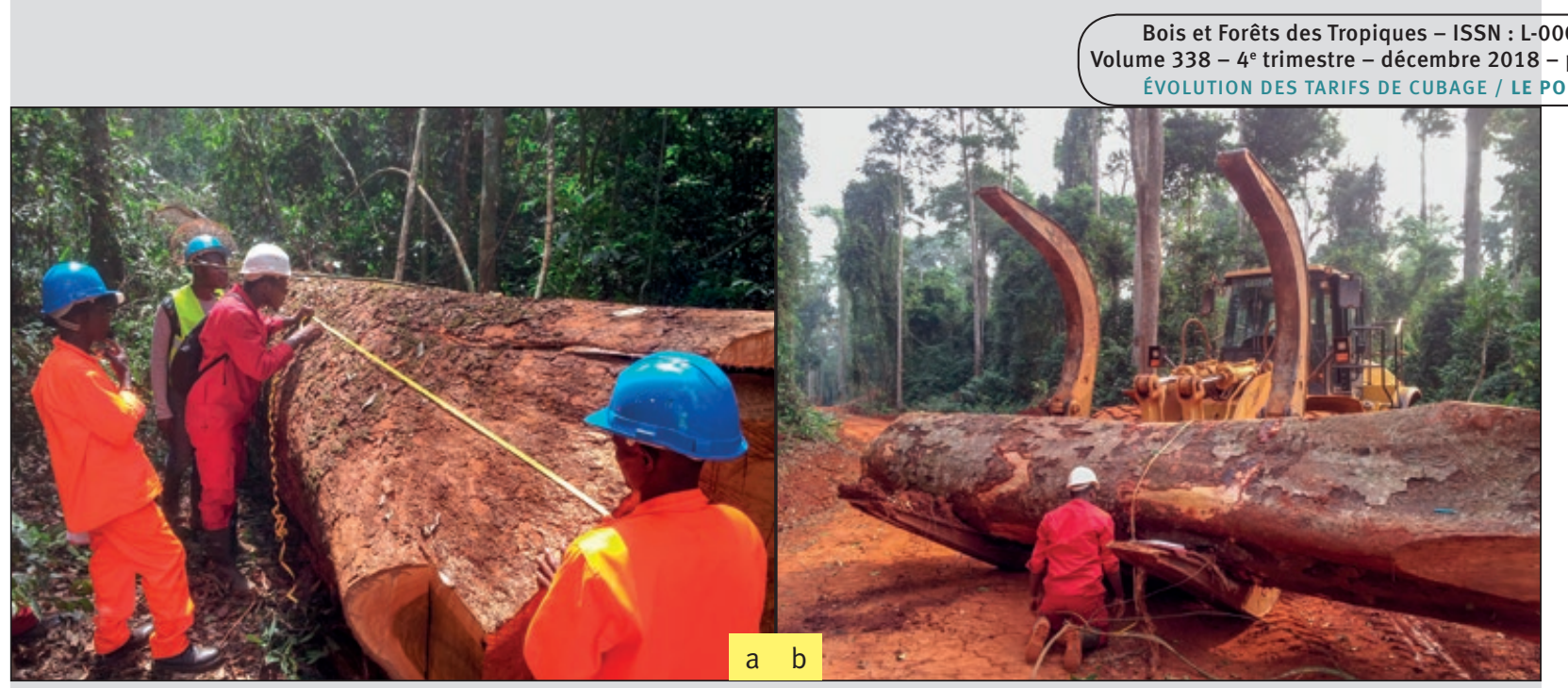

Photos 2.

Estimation du volume par la méthode des billons successifs : a) mesure de la longueur de la bille et $b$ ) mesure du diamètre des billons.

Photos N. Dubart.

Une première phase de prospection a permis d'identifier des arbres bien répartis sur la gamme de diamètres (tableau I) avec 5 tiges par classe de diamètre de $10 \mathrm{~cm}$ d'amplitude et un minimum de 30 tiges par essence. Cette dernière limite correspond à la recommandation empirique de Rondeux (1999), bien qu'elle concernait plutôt des peuplements et des conditions environnementales plus homogènes. Afin de respecter la législation camerounaise qui interdit l'abattage des arbres en dessous du diamètre minimum d'aménagement (DMA), les arbres ayant un diamètre inférieur au DMA ont dans un premier temps été prélevés lors de l'ouverture et de la réhabilitation des routes. Dans un second temps, un permis d'exploitation pour la récolte des produits forestiers à des fins scientifiques a été délivré par l'administration forestière camerounaise. Malgré cela, sur l'ensemble des 732 arbres échantillonnés, avec entre 33 et 117 arbres par essence (figure 2), l'échantillonnage des arbres en dessous du DMA est resté limité à l'issue de la campagne de collecte de données, en particulier pour l'azobé (DMA $=60 \mathrm{~cm})$, le movingui $(D M A=60 \mathrm{~cm}$ ) et les deux talis $(D M A=50 \mathrm{~cm})$. Plus de 80 individus ont cependant été échantillonnés pour l'okan $(n=89)$, le tali suaveolens $(n=92)$, l'ayous $(n=106)$ et le sapelli $(n=117)$. En outre, deux ayous et trois okans de très grandes dimensions (D > $200 \mathrm{~cm}$ ) ont été retirés du jeu de données. Le jeu de données ne contenait en effet pas assez d'individus de plus de $200 \mathrm{~cm}$. Dès lors, nous avons préféré restreindre le jeu de données et la validité des modèles ajustés aux classes de diamètre inférieur à $200 \mathrm{~cm}$, pour lesquelles nous avions suffisamment d'observations. L'amplitude des diamètres échantillonnés et le volume maximum observé sont variables entre essences. Les volumes les plus importants, pouvant atteindre plus de $30 \mathrm{~m}^{3}$, ont été observés pour l'ayous, l'okan et le sapelli (tableau I).

\section{Cubage par billons successifs}

Le diamètre de référence (à hauteur de poitrine, c'està-dire à $1,30 \mathrm{~m}$, ou $30 \mathrm{~cm}$ au-dessus des contreforts/déformations, le cas échéant) a été mesuré avec un ruban diamétrique, et la hauteur de mesure du diamètre a été matérialisée à la peinture. Après l'abattage, la limite de la bille (première grosse branche) a également été marquée à la peinture. Sur les parcs d'exploitation, le volume exploité (c'est-à-dire le volume sur écorce de la bille) a été estimé par la méthode des billons successifs (Rondeux, 1999 ; Fayolle et al., 2013). La longueur de la bille a tout d'abord été mesurée avec une chevillière (photo $2 \mathrm{a}$ ). Les longueurs des billons, un mètre au début de la bille puis deux mètres par la suite quand la bille devient plus régulière, ont ensuite été délimitées à la craie (photo $2 b$ ). Les diamètres gros bout et petit bout de chaque billion ont enfin été mesurés au ruban diamétrique.

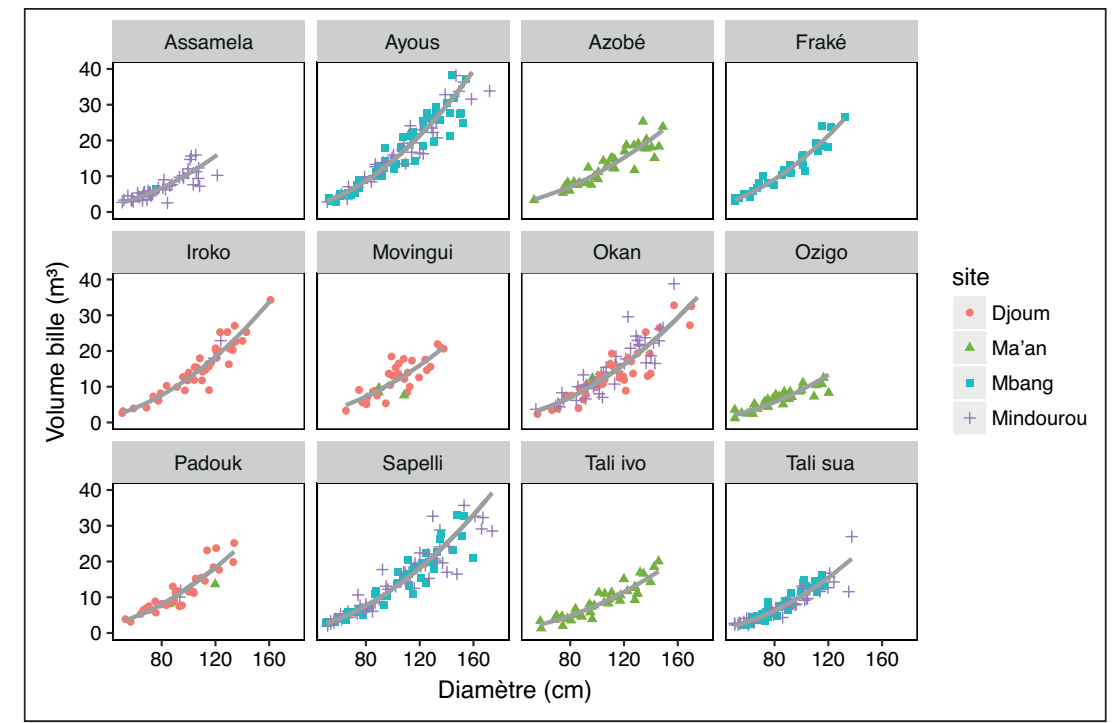

Figure 2.

Relations modélisées entre le volume de la bille exploitable (en $\mathrm{m}^{3}$ ) et le diamètre (en $\mathrm{cm}$ ) des arbres. Les points indiquent les observations (volumes mesurés) par essence et par site (couleur et symbole). Les courbes illustrent par ailleurs les prédictions des tarifs de cubage (volumes ajustés) présentés dans le tableau II. 
L'ensemble de la collecte des données, à l'exception du site de Mindourou, a été réalisé par le même ingénieur forestier. Des contrôles réguliers ont été effectués (près de $20 \%$ des billes ont été remesurées par un second opérateur) et n'ont pas révélé de différence significative entre opérateurs.

\section{Traitement des données}

Le volume sur écorce de chaque billon a été calculé avec la formule du tronc de cône (équation 1) recommandée par Palm (1982) et Rondeux (1999). Le volume de chaque bille, c'est-à-dire la somme de tous les billons correspondants, a ensuite été calculé. Seul le volume sur écorce de la bille exploitable est pris en considération dans cette étude, c'està-dire le volume compris entre le trait de coupe d'abattage (ou de tronçonnage au niveau de la culée) et le trait de coupe de tronçonnage au niveau de la première grosse branche.

$$
V_{j}=\frac{\pi L_{j}}{12}\left(D G B_{j}^{2}+D F B_{j}^{2}+D G B_{j} \cdot D F B_{j}\right) \quad \text { équation } 1
$$

avec $V_{j}$ le volume du billon $j, D G B_{j}$ et $D F B_{j}$ les diamètres gros et fin bout du billon et $L_{j}$ la longueur du billon.

Étant donné l'absence de mesure de hauteur d'arbre ou de longueur de bille dans les inventaires au Cameroun, seuls des tarifs de cubage à une entrée ont été ajustés aux données. Ce sont donc des modèles prédisant le volume sur écorce de la bille exploitable $(V)$ à partir uniquement du diamètre de référence $\left(D_{j}\right)$. Pour chaque essence, quatre modèles polynomiaux ont été testés (équations 2 à 5), ainsi qu'un modèle puissance avec (équation 6) et sans terme additif (équation 7).

$$
\begin{aligned}
& V_{i}=c D_{i}^{2}+\varepsilon_{i} \\
& V_{i}=\mathrm{a}+\mathrm{c} \mathrm{D}_{\mathrm{i}}^{2}+\varepsilon_{\mathrm{i}} \\
& V_{i}=\mathrm{b} \mathrm{D}+\mathrm{c} \mathrm{D}_{\mathrm{i}}^{2}+\varepsilon_{\mathrm{i}} \\
& V_{i}=\mathrm{a}+\mathrm{b} \mathrm{D}+\mathrm{c} \mathrm{D}_{\mathrm{i}}^{2}+\varepsilon_{\mathrm{i}} \\
& V_{i}=\mathrm{a}+\mathrm{b} \mathrm{D}_{\mathrm{i}}^{\mathrm{c}}+\varepsilon_{\mathrm{i}} \\
& V_{i}=\mathrm{b} \mathrm{D}_{\mathrm{i}}^{\mathrm{c}}+\varepsilon_{\mathrm{i}}
\end{aligned}
$$

équation 2 équation 3 équation 4 équation 5 équation 6 équation 7 compris la modélisation de la variance résiduelle), c'est-àdire le meilleur tarif de cubage, pour chaque essence. Il s'agit notamment du critère d'information d'Akaike (AIC) et de l'erreur quadratique moyenne (RMSE, pour Root Mean Square Error, en $\mathrm{m}^{3}$, équation 10), pour l'ensemble des modèles. La structure des résidus a également été analysée graphiquement, et prise en compte, pour choisir le meilleur modèle.

$$
R M S E=\sqrt{\frac{\sum_{i}\left(V_{\mathrm{i}}-\hat{V}_{\mathrm{i}}\right)^{2}}{n}}
$$

équation 10

avec $V_{i}$ le volume de l'arbre $i$ mesuré par la méthode des billons successifs, $\hat{V}_{\mathrm{i}}$ l'estimation du volume du même arbre $i$ par le tarif de cubage évalué et $n$ le nombre d'arbres.

Une fois le meilleur modèle identifié pour une essence donnée, une approche de validation croisée de type «leave one out » a également été réalisée. II s'agit de comparer la performance du modèle ajusté sur l'ensemble du jeu de $n$ données aux modèles ajustés sur des sous-jeux de $n-1$ données (ensemble des données moins une observation). Cette procédure est ensuite répétée autant de fois qu'il y a d'arbres dans le jeu de données ( $n$ fois) (Venables et Ripley, 2013).

Afin de comparer les différents tarifs de cubage, le biais a été calculé (BIAIS, équation 11) et sa significativité a été évaluée à l'aide du test T de Student sur échantillons appariés. L'erreur relative (ER \%, équation 12) et l'efficience de modélisation (EFF, équation 13) ont également été calculées. L'efficience de modélisation s'interprète comme le coefficient de détermination $\left(R^{2}\right)$. Si le tarif de cubage s'ajuste bien aux données observées, l'efficience de modélisation sera élevée et de maximum $100 \%$. À la différence du coefficient de détermination, l'efficience de modélisation peut par contre devenir négative si le modèle est fortement biaisé. Enfin, afin de tester si l'éventuel biais diminue ou augmente avec la taille des arbres, nous avons ajusté des modèles linéaires entre les résidus $\left(\hat{V}_{i}-V_{\mathrm{j}}\right)$ et le diamètre mesuré et testé si les coefficients (soit $a$ pour l'ordonnée à l'origine et $b$ pour la pente) de ces modèles étaient statistiquement différents de 0 .

où $V_{i}$ est le volume bille sur écorce $\left(\mathrm{m}^{3}\right)$ de l'arbre $i, D_{i}$ le diamètre de référence $(\mathrm{cm}), a, b$ et $c$ les paramètres à ajuster et $\varepsilon_{i}$ les résidus du modèle de distribution normale centrée et de variance résiduelle $\operatorname{var}\left(\varepsilon_{\text {p }}\right)$.

Les modèles linéaires et le modèle puissance ont été ajustés par la méthode des moindres carrés. Étant donné l'hétéroscédasticité des résidus marquée par une augmentation attendue de la variance des volumes mesurés avec le diamètre (Picard et al., 2012), deux approches ont été prises en compte pour modéliser la variance résiduelle : en fonction d'une relation linéaire (équation 8) ou non linéaire (équation 9) entre cette variance résiduelle et le carré du diamètre de référence.

$$
\begin{aligned}
& \operatorname{var}\left(\varepsilon_{i}\right)=\sigma^{2} D_{i}^{2} \quad \text { équation } 8 \\
& \operatorname{var}\left(\varepsilon_{i}\right)=\sigma^{2}\left(D_{i}^{2}\right)^{2 \delta} \quad \text { équation } 9
\end{aligned}
$$

où $\sigma^{2}$ et $\delta$ sont les paramètres d'échelle et de forme à ajuster. Différents critères ont été considérés pour sélectionner le meilleur modèle (y

D ans l'article, par souci de clarté, les notations suivantes sont utilisées pour bien distinguer les différents volumes :

- «Volumes mesurés » fait référence aux volumes sur écorce de la bille exploitée obtenus par la méthode des billons successifs.

- "Volumes ajustés » fait référence aux volumes prédits par les équations de cubage ajustées dans cette étude.

- «Volumes GlobAllomeTree » fait référence aux volumes estimés à partir des équations de cubage à une entrée disponible dans la plateforme GlobAllomeTree pour les essences étudiées.

- «Volumes TIAMA » fait référence aux volumes estimés à partir des équations de cubage imposées par l'administration camerounaise et implémentées dans le logiciel TIAMA.

- « Volumes DF10 » fait référence aux volumes estimés par cubage global en appliquant la formule du volume d'un cylindre de diamètre égal à la moyenne des diamètres fin bout et gros bout, et de longueur égale à la longueur de la bille exploitée (équation 14). C'est la méthode appliquée pour cuber les billes sur les parcs d'exploitation en vue de renseigner les volumes exploités dans les documents sécurisés (DF10) de l'administration forestière camerounaise. 


$$
\begin{aligned}
& B I A I S=E\left(\hat{V}_{i}\right)-E\left(V_{i}\right) \\
& E R \%=100 \cdot \frac{E\left(\hat{V}_{i}-V_{i}\right)}{V_{i}} \\
& E F F=100 \cdot \frac{\left(1-\sum_{i}\left(V_{i}-\hat{V}_{i}\right)^{2}\right)}{\sum_{i}\left(E\left(V_{i}\right)-\hat{V}_{i}\right)^{2}}
\end{aligned}
$$

équation 12

équation 13

où $V_{i}$ est le volume mesuré par billon successif de l'arbre $i$,

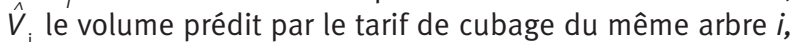
$n$ le nombre d'observations et $E\left(V_{\mathrm{i}}\right)$ la moyenne des volumes observés. Le biais est donc positif si l'estimation surestime le volume.

Pour chaque arbre échantillonné, les volumes sur écorce de la bille ont également été estimés avec les tarifs de cubage de l'administration forestière du Cameroun. Les tarifs de cubage de l'administration sont définis par espèce et par zone géographique appelée " phase ». Pour effectuer nos comparaisons, nous avons utilisé, pour chaque arbre les tarifs de cubage de l'essence et de la phase adéquate. Pour appréhender le biais entre les tarifs ajustés et les tarifs de l'administration, des facteurs de correction des volumes calculés avec les tarifs de l'administration ont été calculés pour les essences et les phases étudiées. Ce coefficient a été calculé en faisant le rapport entre les volumes ajustés et les volumes calculés avec les tarifs de l'administration.

Le volume de la bille de chaque arbre échantillonné a aussi été estimés avec l'ensemble des tarifs de cubage à une entrée disponibles dans la plateforme GlobAllomeTree (Henry et al., 2013) pour les 12 essences étudiées. Enfin, le volume des billes a également été calculé à l'aide de l'équation 14 en utilisant les mesures des diamètres fin bout et gros bout ainsi que la longueur de la bille. Cette dernière méthode est la méthode de cubage global employée sur les parcs pour l'obtention des volumes exploités et inscrits dans les documents sécurisés de l'administration appelés DF10.

$$
V_{\mathrm{i}}=\left(\frac{D G B_{\mathrm{i}}+D F B_{\mathrm{i}}}{4}\right)^{2} \pi L_{i}
$$

équation 14

L'ensemble des analyses statistiques a été réalisé avec le logiciel libre R version 3.3.2 (R Core Team, 2016), et en particulier avec le package nlme version 3.1-128, pour l'ajustement des modèles par la méthode des moindres carrés généralisés (Pinheiro et al., 2012).

\section{Résultats}

\section{Ajustement et validation des modèles}

Pour 11 essences sur 12 étudiées, le meilleur modèle sélectionné sur la base de l'AIC et de la RMSE est le modèle de l'équation $4, V=b D+c D^{2}$ (tableau II). Pour le sapelli, le modèle de l'équation $5, V=a+b D+c D^{2}$, qui inclut une ordonnée à l'origine, apparaît légèrement meilleur. Pour toutes les essences, la modélisation de la variance résiduelle avec l'approche non linéaire (équation 8) a fourni de meilleurs résultats et a donc été préférée. La dispersion des résidus standardisés de ces modèles montre que leur hétéroscédasticité a été correctement prise en compte et que les modèles ne présentent pas de biais sur l'ensemble des gammes de diamètres étudiées (annexe 1). Les performances des modèles sont néanmoins variables, avec une RMSE de 1,33 $\mathrm{m}^{3}$ pour l'ozigo et pouvant aller jusqu'à $3,47 \mathrm{~m}^{3}$ pour l'ayous. Cette valeur très élevée s'explique par des volumes mesurés extrêmement importants et fréquemment au-delà de $30 \mathrm{~m}^{3}$ pour cette essence (figure 2).

Tableau II.

Résultats des ajustements des modèles sélectionnés et prédisant le volume exploitable de la bille sur écorce $\left(\mathrm{m}^{3}\right)$

\begin{tabular}{|c|c|c|c|c|c|c|c|}
\hline Essence & Modèle & a & b & C & $\delta$ & $\sigma$ & RMSE \\
\hline Assamela & équation 4 & & $-6,558 \mathrm{e}-03$ & $1,136 \mathrm{e}-03$ & 1,19 & $4,56 \mathrm{e}-05$ & 1,97 \\
\hline Ayous & équation 4 & & $-2,87 e-02$ & $1,724 \mathrm{e}-03$ & 1,22 & $2,91 \mathrm{e}-05$ & 3,47 \\
\hline Azobé & équation 4 & & $1,43 \mathrm{e}-02$ & $9,345 \mathrm{e}-04$ & 1,26 & $1,36 \mathrm{e}-05$ & 2,42 \\
\hline Fraké & équation 4 & & $-1,317 \mathrm{e}-02$ & $1,591 \mathrm{e}-03$ & 1,04 & $1,29 \mathrm{e}-04$ & 1,44 \\
\hline Iroko & équation 4 & & $-1,998 \mathrm{e}-02$ & $1,441 \mathrm{e}-03$ & 1,20 & $2,95 e-05$ & 2,87 \\
\hline Movingui & équation 4 & & $5,072 \mathrm{e}-03$ & $1,067 \mathrm{e}-03$ & 0,53 & $2,32 \mathrm{e}-02$ & 2,94 \\
\hline Okan & équation 4 & & $-7,940 \mathrm{e}-03$ & $1,194 \mathrm{e}-03$ & 1,14 & $6,88 e-05$ & 3,39 \\
\hline Ozigo & équation 4 & & $-8,48 e-03$ & $9,938 \mathrm{e}-04$ & 0,83 & 8,47e-04 & 1,33 \\
\hline Padouk & équation 4 & & $-1,372 \mathrm{e}-03$ & $1,28 \mathrm{e}-03$ & 1,14 & $6,38 e-05$ & 2,10 \\
\hline Sapelli & équation 5 & $-2,793 e-01$ & $-1,005 e-02$ & $1,363 \mathrm{e}-03$ & 1,16 & $5,27 e-05$ & 3,23 \\
\hline Tali ivo & équation 4 & & $-1,019 e-02$ & $8,825 e-04$ & 0,61 & $5,76 \mathrm{e}-03$ & 1,71 \\
\hline Tali sua & équation 4 & & $-2,354 \mathrm{e}-02$ & $1,27 e-03$ & 1,04 & $1,27 \mathrm{e}-04$ & 1,68 \\
\hline
\end{tabular}
en fonction du diamètre de référence $(\mathrm{cm})$ pour les 12 essences commerciales étudiées. La valeur des paramètres ajustés $a, b, c, \delta$ et $\sigma$ est indiquée. L'erreur associée à chaque paramètre ainsi que les corrélations entre ces paramètres (matrices de variance-covariance) sont présentées en annexe (annexe 3). 
Tableau III.

Évaluation de la précision et du biais des volumes ajustés, des tarifs de cubage utilisés par l'administration camerounaise (volumes TIAMA) et des volumes estimés sur parc par cubage global (volumes DF10). La qualité de ces méthodes de cubage est appréciée à l'aide du biais (équation 12), de l'efficience de modélisation (EFF, équation 13), de la RMSE (équation 10, en $\mathrm{m}^{3}$ ), de l'erreur relative (ER\%, équation 12), des paramètres de la régression entre les erreurs et le diamètre des arbres ( $a$ et $b$ ) ainsi que par le coefficient de détermination de cette dernière relation $\left(R^{2}\right.$, en \%). Le degré de significativité des paramètres ajustés $a, b$ et c est indiqué, avec * pour : $P<0,05$; ${ }^{\star *}$ pour $\mathrm{P}<0,01 ;{ }^{\star \star \star}$ pour $\mathrm{P}<0,001$; et n.s. pour « non significatif ».

\begin{tabular}{|c|c|c|c|c|c|c|c|c|}
\hline Cubage & Essence & Biais & EFF & RMSE & ER\% & a & b & $\mathbf{R}^{\mathbf{2}}$ \\
\hline \multirow[t]{12}{*}{ Volumes ajustés } & Assamela & 0,160 n.s. & 70,6 & 2,021 & 21,3 & $-1,040$ n.s. & 0,016 n.s. & 3,2 \\
\hline & Ayous & 0,166 n.s. & 90,2 & 3,506 & 13,8 & $-1,259$ n.s. & 0,014 n.s. & 1,9 \\
\hline & Azobé & 0,197 n.s. & 83,2 & 2,533 & 13,5 & - 2,733 n.s. & 0,027 n.s. & 7,9 \\
\hline & Fraké & 0,043 n.s. & 94,4 & 1,514 & 10,3 & $-0,245$ n.s. & 0,004 n.s. & 0,3 \\
\hline & Iroko & $-0,032$ n.s. & 88,1 & 2,966 & 13,4 & 0,009 n.s. & 0,000 n.s. & 0,0 \\
\hline & Movingui & 0,026 n.s. & 46,0 & 3,088 & 24,0 & $-0,646$ n.s. & 0,007 n.s. & 0,2 \\
\hline & Okan & $-0,077$ n.s. & 79,1 & 3,445 & 19,9 & 0,356 n.s. & 0,004 n.s. & 0,1 \\
\hline & Ozigo & 0,094 n.s. & 85,1 & 1,392 & 22,5 & - 0,999 n.s. & 0,015 n.s. & 7,8 \\
\hline & Padouk & 0,036 n.s. & 80,6 & 2,210 & 15,4 & $-0,048$ n.s. & 0,001 n.s. & 0,0 \\
\hline & Sapelli & 0,166 n.s. & 89,5 & 3,295 & 16,1 & $-1,051$ n.s. & 0,013 n.s. & 2,1 \\
\hline & Tali ivo & $-0,01$ n.s. & 83,0 & 1,792 & 22,4 & 0,308 n.s. & $-0,003$ n.s. & 0,2 \\
\hline & Tali sua & 0,021 n.s. & 84,0 & 1,732 & 16,5 & $-0,353$ n.s. & 0,004 n.s. & 0,3 \\
\hline \multirow[t]{12}{*}{ Volumes TIAMA } & Assamela & $-0,997 * \star \star *$ & 60,7 & 2,15 & 29,9 & $-0,792$ n.s. & $-0,003$ n.s. & 0,1 \\
\hline & Ayous & $-4,177^{* \star *}$ & 65,5 & 5,90 & 24,3 & 2,441 * & $-0,064 * \star \star$ & 28,9 \\
\hline & Azobé & $-2,493 * \star \star$ & 66,8 & 3,39 & 21,3 & $-1,93$ n.s. & $-0,005$ n.s. & 0,4 \\
\hline & Fraké & 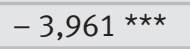 & 34,4 & 4,70 & 38,7 & 2,748 ** & $-0,082 * \star \star$ & 67,2 \\
\hline & Iroko & 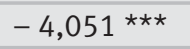 & 45,8 & 5,48 & 23,7 & 4,666 ** & 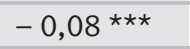 & 38,9 \\
\hline & Movingui & $-3,646 * \star \star *$ & $-24,9$ & 4,93 & 29,2 & 5,129 n.s. & $-0,086^{\star \star}$ & 22,6 \\
\hline & Okan & $-2,710^{\star \star \star \star}$ & 59,6 & 4,42 & 23,4 & $-1,779$ n.s. & $-0,042^{\star \star \star}$ & 14,3 \\
\hline & Ozigo & $-1,357 * \star \star$ & 44,1 & 1,99 & 28,0 & 1,142 * & $-0,035^{\star \star \star}$ & 37,6 \\
\hline & Padouk & $-3,423 * \star \star$ & 19,0 & 4,33 & 30,0 & 3,261 * & $-0,074 * \star \star$ & 35,6 \\
\hline & Sapelli & $-3,035 * \star \star$ & 63,9 & 4,86 & 22,7 & $2,363 * \star$ & $-0,056 * \star \star$ & 31,8 \\
\hline & Tali ivo & 0,743 ** & 83,2 & 1,86 & 25,8 & 0,499 n.s. & 0,002 n.s. & 0,1 \\
\hline & Tali sua & $-0,844^{\star \star \star *}$ & 67,8 & 2,08 & 17,1 & 1,847 * & $-0,032 * \star \star *$ & 12,9 \\
\hline \multirow[t]{12}{*}{ Volumes DF10 } & Assamela & 0,853 *** & 88,9 & 1,211 & 14,5 & 0,103 n.s. & 0,010 n.s. & 4,2 \\
\hline & Ayous & 0,535 n.s. & 93,2 & 3,098 & 13,1 & 0,039 n.s. & 0,005 n.s. & 0,3 \\
\hline & Azobé & 0,521 ** & 95,7 & 1,222 & 7 & 0,395 n.s. & 0,001 n.s. & 0,1 \\
\hline & Fraké & $-0,254$ n.s. & 91,1 & 2,188 & 15,7 & $-1,908$ n.s. & 0,02 n.s. & 5,5 \\
\hline & Iroko & 0,604 * & 95,5 & 1,998 & 9,5 & $-1,028$ n.s. & 0,015 n.s. & 5,1 \\
\hline & Movingui & 0,537 n.s. & 91,4 & 1,777 & 11,5 & 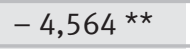 & $0,05^{\star \star}$ & 29,3 \\
\hline & Okan & 1,189 *** & 91,6 & 2,678 & 10,1 & $-0,198$ n.s. & 0,013 n.s. & 2,9 \\
\hline & Ozigo & $0,412^{\star \star \star}$ & 95,8 & 0,771 & 11,4 & $-0,704 * \star$ & $0,015^{\star \star *}$ & 37,1 \\
\hline & Padouk & 0,317 n.s. & 90,8 & 1,799 & 10,2 & $-0,465$ n.s. & 0,009 n.s. & 1,1 \\
\hline & Sapelli & 1,422 *** & 92,8 & 2,806 & 14,8 & $-0,219$ n.s. & $0,017^{\star \star}$ & 6,7 \\
\hline & Tali ivo & 0,004 n.s. & 96,7 & 0,913 & 7,1 & $-0,535$ n.s. & 0,005 n.s. & 2,5 \\
\hline & Tali sua & $-0,356 * \star$ & 91,5 & 1,043 & 9,8 & 1,045 * & $-0,017 * \star$ & 11,8 \\
\hline
\end{tabular}


La validation croisée des modèles ajustés confirme la bonne qualité des ajustements déjà identifiée par l'examen visuel des résidus (tableau III). Ni les biais, ni les coefficients de la régression entre les erreurs et le diamètre des arbres ne sont significativement différents de zéro, et ce pour aucune essence. L'efficience de modélisation est généralement élevée et supérieure à $70 \%$, sauf pour le movingui dont le nombre d'individus $(\mathrm{n}=33)$ et l'amplitude des diamètres observés $(D=65-137 \mathrm{~cm})$ étaient limités.

\section{Comparaison avec les autres méthodes de cubage}

\section{Volumes estimés à partir des tarifs de cubage disponibles dans GlobAllomeTree}

Parmi l'ensemble des tarifs de cubage recensés sur la plateforme GlobAllomeTree, 57 tarifs de cubage disponibles pour les essences étudiées ont été retenus (annexe 2). Ce sont des tarifs de cubage à une entrée, fonction uniquement du diamètre de référence, prédisant le volume de la bille commercialisable sur ou sous écorce. De plus amples informations sur ces tarifs de cubage sont disponibles dans l'annexe 2 ainsi que l'ensemble des indicateurs de précision et de biais utilisés pour évaluer les prédictions. À l'exception de trois tarifs de cubage pour l'azobé développés pour la Côte d'Ivoire et le Cameroun, on observe que la majorité des tarifs de cubage testés sous-estiment les volumes mesurés dans cette étude, c'est-à-dire obtenus par la méthode des billons successifs. Ainsi, parmi les tarifs de cubage sélectionnés, 37 (73\%) sous-estiment significativement les volumes mesurés et seulement $3(6 \%)$ tarifs de cubage les surestiment significativement (figure 3 ). On note également que les biais observés sont généralement du même ordre de grandeur que ceux obtenus à partir des tarifs de cubage utilisés par l'administration camerounaise (volumes TIAMA).

\section{Volumes estimés avec les tarifs de cubage de l'administration (volumes TIAMA)}

La comparaison des volumes mesurés et des volumes estimés avec les tarifs de cubage utilisés par l'administration camerounaise (volumes TIAMA) met en évidence la présence d'un biais significatif pour toutes les essences (figure 4). Ce biais est néanmoins de signe et d'ampleur variables (tableau III). Pour le tali ivorense, le biais est faible et positif alors que pour toutes les autres essences le biais est négatif (et plus important). Pour ces autres essences, le biais moyen varie de $-1,0 \mathrm{~m}^{3}$ pour l'assamela à $-4,1 \mathrm{~m}^{3}$ pour l'ayous. En outre, ce biais augmente, en valeur absolue, avec la taille des individus (paramètre $b$ significatif dans le tableau III) pour la majorité des essences à l'exception de l'assamela, de l'azobé et du tali ivorense. L'efficience de modélisation est la plus élevée pour le tali ivorense (83\%) et est particulièrement faible pour le movingui (-25\%, tableau III). L'erreur relative de ces tarifs de cubage sur les données mesurées est comprise entre $17 \%$ (tali suaveolens) et $30 \%$ (padouk).

\section{Volumes estimés sur parc (volumes DF10)}

La comparaison des volumes obtenus par la méthode des billons successifs (volumes mesurés) et par la méthode de cubage global employée sur les parcs d'exploitation (volumes DF10) montre que l'efficience de modélisation est très élevée et, comme l'on pouvait s'y attendre, supérieure à celle obtenue à l'aide des tarifs de cubage (tableau III). Les volumes DF10 sont donc généralement très proches des volumes mesurés. Néanmoins, pour certaines essences, un biais significatif est détecté (de 0,5 à $1,4 \mathrm{~m}^{3}$ ). Un biais positif est notamment significatif pour 6 des 12 essences étudiées, à savoir l'assamela, l'azobé, l'iroko, l'okan, l'ozigo et le sapelli. Un biais négatif et significatif, mais relativement faible, a été identifié pour le tali suaveolens.

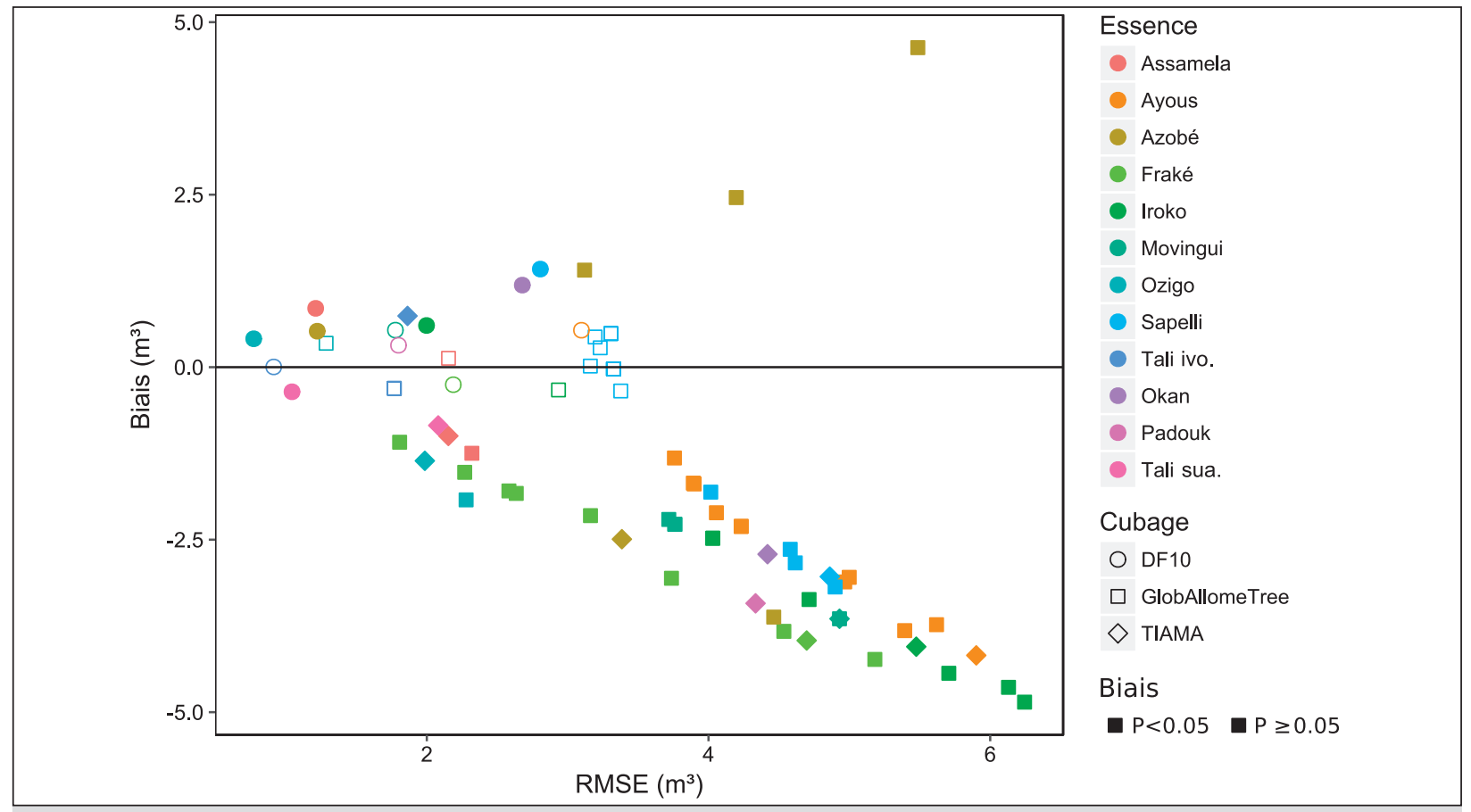

Figure 3.

Comparaison du biais (équation 11) et de la RMSE (équation 10) des volumes estimés par les tarifs de l'administration camerounaise (volumes TIAMA), avec la formule de cubage global utilisée pour cuber les billes sur parc (volumes DF10) et avec une sélection des tarifs disponibles dans la plateforme GlobAllomeTree (volumes GlobAllomeTree). Un symbole plein indique un biais significatif. 


\section{Domaine de validité}

Afin de limiter les biais liés à l'utilisation des tarifs de cubage proposés, il convient de ne pas les utiliser en dehors de leur domaine d'application. Ces tarifs de cubage ne peuvent rigoureusement être utilisés que pour les combinaisons d'essences, de sites et de diamètres représentées dans l'échantillon (tableau I et figure 2). L'effort d'échantillonnage devrait être poursuivi afin d'obtenir des tarifs de cubage encore plus robustes et représentatifs de l'ensemble des conditions rencontrées au Cameroun.

Dans notre étude, ce sont 732 arbres qui ont été utilisés, avec des effectifs par essence compris entre 33 (movingui) et 117 (sapelli). Ces effectifs sont plus faibles que ceux utilisés dans les études menées en forêt tempérée. Rondeux (1999) recommande, à ce titre, d'échantillonner entre 30 et 100 arbres pour établir un tarif de cubage pour un peuplement homogène où tous les arbres ont été sou-

Coefficient de conversion des volumes TIAMA pour obtenir les volumes estimés à partir des nouveaux tarifs de cubage (volumes ajustés). Les tarifs de cubage TIAMA sont définis par région géographique (appelée phase).

\section{Discussion}

L'objectif général de cette étude était d'apporter une réflexion sur la nécessité de réviser les tarifs de cubage en vigueur au Cameroun, comme suggéré par plusieurs études (Fayolle et al., 2013 ; Tchatat et al., 2008), à partir de l'échantillonnage destructif des principales essences commerciales. À partir du plus grand jeu de données destructives collectées en Afrique centrale ( $n=732$ arbres) et couvrant une large gamme de diamètres (jusqu'à $190 \mathrm{~cm}$ ) et de volumes (jusqu'à $47 \mathrm{~m}^{3}$ ), nous avons pu ajuster des équations allométriques du volume exploitable spécifique pour 12 essences. Pour chaque essence étudiée, les tarifs de cubage élaborés sont d'une précision satisfaisante avec une erreur moyenne pour l'estimation du volume exploitable d'un arbre comprise entre 1,5 $\mathrm{m}^{3}$ (fraké) et 3,5 $\mathrm{m}^{3}$ (ayous) et une erreur moyenne relative comprise entre $10,3 \%$ (fraké) et $24,0 \%$ (movingui). La précision des tarifs de cubage aurait été notamment meilleure en ajoutant la longueur de la bille comme deuxième variable explicative (tarif de cubage à deux entrées) (Rondeux, 1999 ; Akindele et LeMay, 2006) Par exemple, la RMSE d'un tarif de cubage à deux entrées pour l'ayous serait alors réduite à $2,5 \mathrm{~m}^{3}$ (ajustement non présenté). Ceci n'aurait toutefois qu'un intérêt purement théorique car les longueurs de bille ne sont pas mesurées lors des inventaires d'aménagement et d'exploitation. mis à une même sylviculture et aux mêmes conditions écologiques. Certaines études utilisent dès lors parfois plusieurs milliers d'individus pour établir des tarifs de cubage pour une seule espèce et ainsi identifier les différentes sources de variabilité des volumes (Zianis et al., 2005). Un tel effort est quasi impossible dans les forêts denses humides naturelles où les espèces commerciales peuvent être très disséminées et présenter des structures de populations complexes et/ou variables (Durrieu de Madron et Forni, 1997). Les données issues de ce travail ont le mérite d'avoir été collectées pendant 960 hommes-jours, dans quatre sites caractérisés par des conditions écologiques contrastées au Cameroun, avec la même méthodologie, par les mêmes personnes et en garantissant un nombre relativement constant d’individus pour chaque classe de diamètre.

\section{Une inadéquation confirmée des tarifs de cubage de l'administration camerounaise}

En accord avec les résultats déjà rapportés au Cameroun pour trois essences par Fayolle et al. (2013), avec néanmoins un nombre limité d'arbres par essence, ces résultats basés sur le plus grand jeu de données destructives collectées en Afrique centrale confirment la sous-estimation des tarifs de cubage implémentés dans le logiciel TIAMA, logiciel imposé par l'administration camerounaise. La nécessité de réviser les tarifs de cubage en vigueur au Cameroun est aujourd'hui incontestable. 


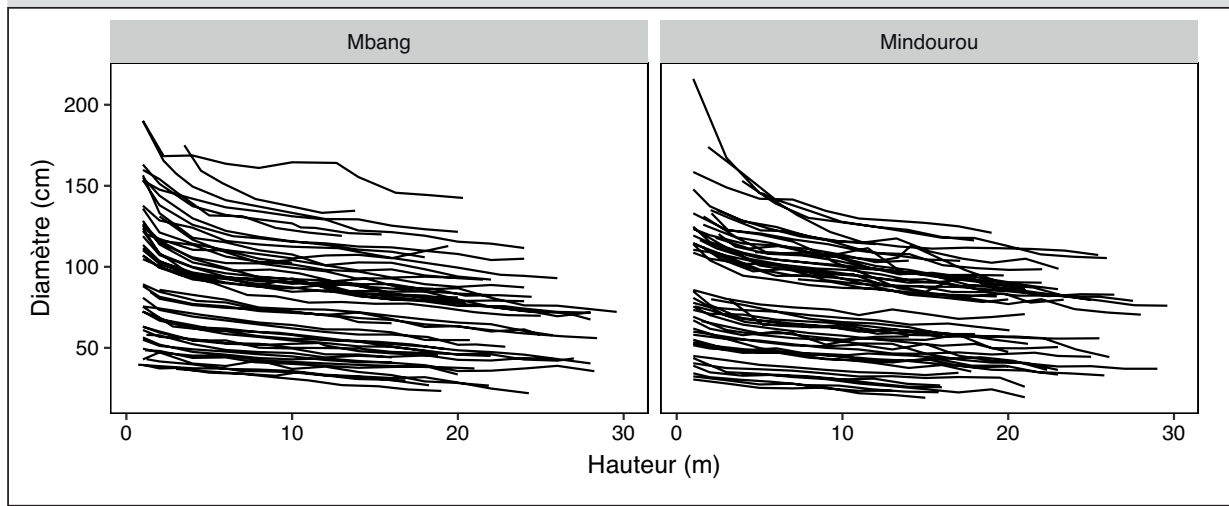

Figure 5.

Variation du diamètre depuis la base de chaque billon de sapelli jusqu'à la hauteur de recoupe dans les sites de Mbang ( $n=49$ arbres) et Mindourou ( $n=68$ arbres). Une ligne correspond à un arbre. On note que la forme de la bille est effectivement légèrement curviligne pour bon nombre d'individus (et surtout pour les individus de grandes dimensions) et peut donc mener à un biais lors de l'utilisation de la formule du cubage global (volumes DF10).
Bois et Forêts des Tropiques - ISSN : L-0006-579X Volume $338-4^{\mathrm{e}}$ trimestre - décembre 2018 - p. 57-71 ÉVOLUTION DES TARIFS DE CUBAGE / LE POINT SUR.

D'une part, lors de l'évaluation de la ressource avant l'exploitation, le volume exploitable est sous-estimé (volumes du logiciel TIAMA). Et d'autre part, après exploitation, le volume exploité est surestimé (volumes DF10). Ces deux biais concourent aux différences observées entre les volumes exploitables et les volumes exploités, menant au constat que le volume prévu par le plan annuel d'opération est régulièrement atteint avec un nombre d'arbres beaucoup plus restreint que celui prévu par le même plan annuel d'opération (Tchatat et al., 2008).

\section{Changement de la ressource ou changement des pratiques d'exploitation?}

En effet, les tarifs de cubage issus du logiciel TIAMA sous-estiment les volumes mesurés par billons successifs pour toutes les essences testées à l'exception du tali ivorense. Dans notre étude, le bais pour le calcul du volume d'un arbre était en moyenne compris entre $0,9 \mathrm{~m}^{3}$ (pour l'assamela) et 4,2 $\mathrm{m}^{3}$ (pour l'ayous) et ce biais augmentait généralement avec la taille des arbres comme l'avaient également souligné Fayolle et al. (2013). En utilisant la figure 3 comme un abaque, les tarifs de cubage ajustés dans cette étude peuvent être utilisés pour corriger ce biais en fonction de l'essence et de la taille des arbres.

Si la justesse des tarifs de cubage de l'administration peut effectivement être remise en question, une deuxième source de biais semble également devoir être prise en considération pour expliquer les différends entre les gestionnaires/exploitants forestiers et l'administration (Tchatat et al., 2008). L'utilisation de la formule de cubage global (équation 14) pour cuber les billes sur les parcs d'exploitation (volumes DF10) lors du contrôle des volumes exploités peut en effet mener à un biais important en fonction de la forme des arbres. Cette formule est notamment biaisée si la forme réelle de la bille s'approche plus d'un néloïde (forme légèrement curviligne), avec un défilement plus élevé à la base de l'arbre (comme on a pu l'observer pour le sapelli, par exemple ; figure 5). Ce dernier biais ne concerne donc vraisemblablement pas toutes les essences et pourrait dépendre de la présence de contrefort. En présence de contrefort, une part importante de la base bille est régulièrement abandonnée et la partie exploitée se concentre sur les parties plus régulières qui présentent une forme plus proche d'un tronc de cône (figure 5). D’après notre jeu de données, les volumes estimés de cette façon seraient notamment peu ou pas biaisés pour l'ayous, le fraké, le padouk et le tali ivorense. Pour les autres essences, ce biais semble moins important que celui lié à l'utilisation du tarif de cubage du logiciel TIAMA. Néanmoins, pour l'okan et le sapelli, les biais moyens pour notre échantillon étaient tout de même de 1,2 et $1,4 \mathrm{~m}^{3}$, soit environ $45 \%$ de la valeur du biais lié à l'utilisation du tarif de cubage du logiciel TIAMA.
Les biais rapportés pour les tarifs de cubage TIAMA et pour la majorité des tarifs de cubage disponibles dans la base de données GlobAllomeTree pourraient avoir différentes origines, notamment liées à la qualité des mesures et/ou l'échantillonnage, à l'évolution de la ressource exploitable, et à l'évolution des pratiques d'exploitation forestière.

Puisque le biais a été observé pour toutes les essences, dans plusieurs sites et en comparant plus de 30 tarifs de cubage résultant de 16 études différentes publiées entre 1962 et 2011, on peut raisonnablement mettre de côté un artéfact lié à la qualité des mesures. Pour expliquer ce biais, on peut faire l'hypothèse que, par rapport aux billes échantillonnées dans les autres tarifs de cubage (TIAMA et GlobAllomeTree), les billes échantillonnées dans cette étude sont en moyenne plus longues et/ou de forme différente. La longueur peut notamment varier avec les pratiques d'exploitation qui visent à optimiser de plus en plus la ressource exploitable étant donné sa raréfaction progressive. En effet, de nombreuses sociétés exploitent actuellement dans des forêts en deuxième ou troisième rotation. Les arbres exploités ont en conséquence des tailles et des qualités de fûts probablement différentes de celles observées lors du premier passage au cours duquel seuls les plus beaux arbres étaient abattus.

En outre, notre étude a été conduite essentiellement dans des concessions certifiées FSC qui mettent en œuvre des pratiques d'exploitation à faible impact visant à réduire les pertes et à optimiser les volumes sortis de la forêt (FAO, 2003). Bien que la nécessité de la mise en œuvre de telles pratiques fasse aujourd'hui partie de l'arsenal juridique de la plupart des pays d'Afrique centrale, leur contrôle est particulièrement effectif dans les concessions certifiées. Les purges, tant en forêt que sur les parcs, y sont en conséquence plus limitées qu'autrefois (Sist, 2000). Ces pratiques conduisent à exploiter des billes de longueur supérieure et à accepter davantage de défauts dans le bois des billes commercialisées. Le marché du bois est lui-même plus souple qu'auparavant et tolère davantage d'imperfections vu les innovations dans les méthodes de transformation, dont l'aboutage. 


\section{Conclusion}

Cette étude confirme l'inadéquation de la plupart des tarifs de cubage existant jusqu'à présent pour évaluer le volume des billes exploitées pour la majorité des essences au Cameroun. Comme connu et suggéré par de nombreux acteurs de la gestion forestière, il apparaît indispensable de réviser les tarifs de cubage des essences exploitées ou potentiellement exploitables au Cameroun. Cette étude propose à ce titre, pour 12 essences commerciales majeures, des nouveaux tarifs de cubage ainsi que des facteurs de correction à appliquer aux tarifs de cubage actuellement imposés par l'administration. Toutefois, bien que cette étude ait été menée dans des sites représentatifs des principaux types forestiers du Cameroun, il conviendrait d'en augmenter encore la robustesse en multipliant les sites d'étude tant en termes de contextes écologiques qu'économiques. En effet, la justesse de ces tarifs de cubage à une entrée est avant tout tributaire de la longueur des billes et donc de pratiques de valorisation propres aux entreprises concernées.

\section{Remerciements}

Cette étude a été réalisée dans le cadre du Programme de promotion de l'exploitation certifiée des forêts (PPECF) au travers du Projet ACPAC, «Amélioration continue des plans d'aménagement au Cameroun », mis en œuvre par l'association belge Nature $^{+}$. Les auteurs remercient les sites et sociétés forestières partenaires du projet ACPAC, les membres du Comité scientifique consultatif de suivi des activités de recherche dans les forêts du domaine forestier permanent, et les participants de la seconde session dudit Comité, du 26 avril 2017, à Yaoundé au Cameroun, pour leur retour constructif.

\section{Financement}

Ce projet a été financé par la Commission des forêts d'Afrique centrale (COMIFAC) dans le cadre du Programme de promotion de l'exploitation certifiée des forêts (PPECF) et mis en œuvre entre juillet 2014 et mai 2017 par l'association belge Nature+ (http://www.natureplus.be/), qui est spécialisée dans la gestion durable des ressources naturelles, et œuvre pour le développement et la recherche appliquée au service des milieux tropicaux.

\section{Annexe 1.}

Résidus (écarts entre les prédictions et les observations) des modèles (tarifs de cubage) ajustés pour chacune des essences étudiées (tableau II). Les lignes verticales présentent la distribution des résidus par classes de valeurs prédites contenant un même nombre de résidus. Les traits fins indiquent la variance des résidus correspondants qui est supposée être constante pour chaque classe et les traits épais indiquent l'intervalle de confiance de la moyenne de ces résidus supposée égale à zéro.

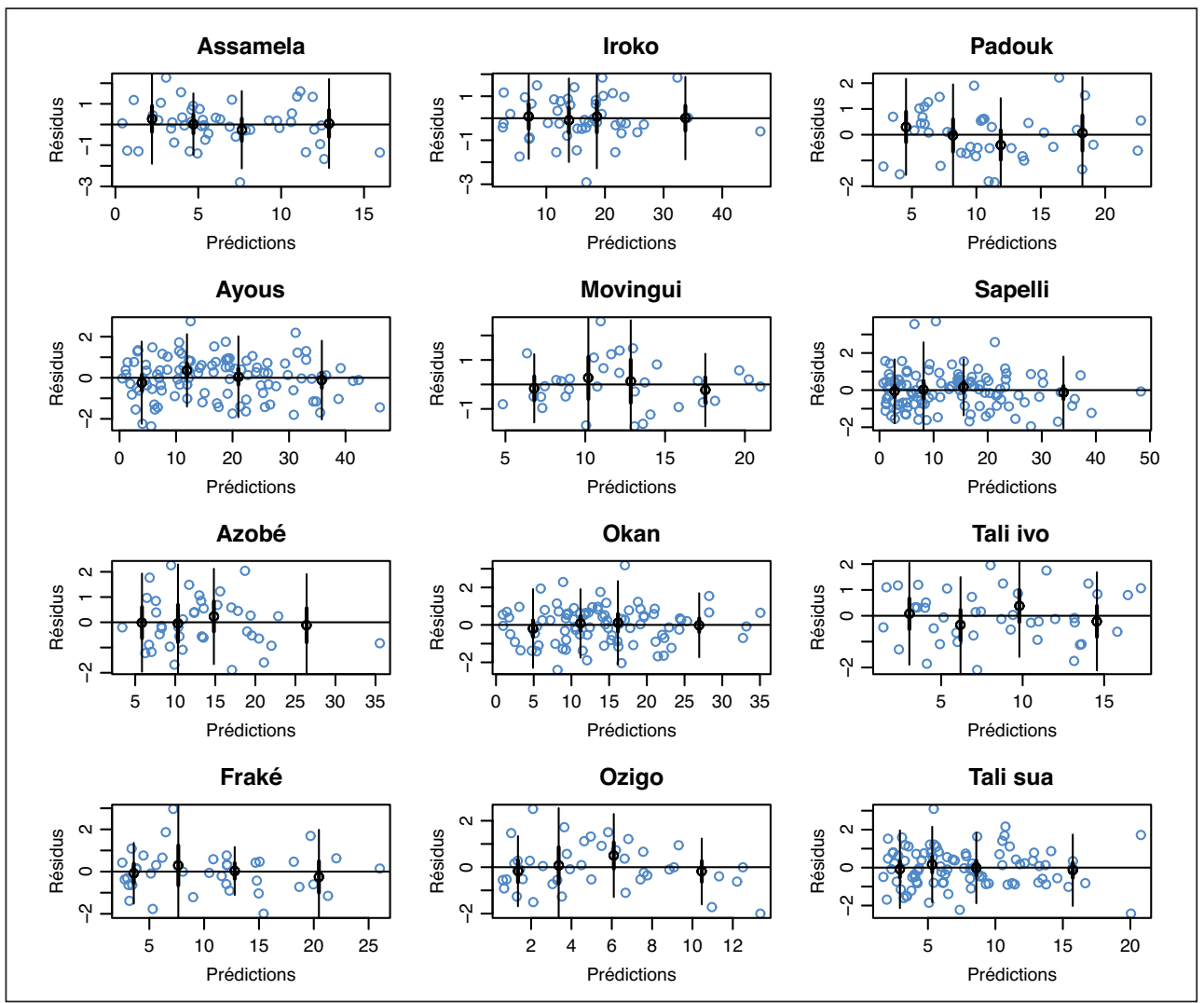




\section{Annexe 2}

Test des prédictions des tarifs de cubage disponibles dans la plateforme GlobAllomeTree (Henry et al., 2013 ; http://www. globallometree.org/) pour les 12 essences étudiées. Pour chaque tarif de cubage, les prédictions ont été confrontées aux données observées (volumes mesurés). Outre l'essence et l'identifiant du tarif de cubage, le biais et sa significativité, l'efficience de modélisation (EFF en \%), la RMSE (en $\mathrm{m}^{3}$ ), l'erreur relative moyenne (ER\%), les paramètres de la régression entre les erreurs et le diamètre des arbres ( $a$ et $b$ ) ainsi que le coefficient de détermination de cette dernière relation $\left(R^{2}\right)$ sont renseignés. Les tarifs de cubage du volume sur écorce sont distingués des tarifs de cubage sous écorce par une croix dans la colonne « Écorce ». Le pays et la référence du tarif de cubage sont indiqués. La significativité des paramètres est indiquée avec: * pour $P<0,05$, ** pour $P<0,01,{ }^{\star \star \star}$ pour $P<0,00$; et n.s. pour « non significatif ».

\begin{tabular}{|c|c|c|c|c|c|c|c|c|c|c|c|}
\hline Essence & Tarif ID & Biais & EFF & RMSE & ER\% & a & b & $\mathbf{R}^{2}$ & Écorce & Pays & Référence \\
\hline Ayous & 46713 & $-1,68^{* \star \star}$ & 85,0 & 3,89 & 17,4 & 0,857 n.s. & $-0,025^{*}$ & 6,0 & $x$ & CMR & API Dimako \\
\hline Fraké & 48670 & $-1,794^{\star \star \star \star}$ & 80,0 & 2,58 & 23,2 & 0,659 n.s. & $-0,03^{*}$ & 16,5 & $x$ & CMR & API Dimako \\
\hline Sapelli & 37426 & 0,487 n.s. & 85,8 & 3,31 & 40,5 & $2,717^{\star \star \star *}$ & $-0,023^{\star \star}$ & 7,3 & $\mathrm{x}$ & CMR & API Dimako \\
\hline Fraké & 47115 & 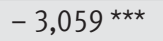 & 55,7 & 3,74 & 29,5 & 2,106 * & $-0,063^{\star \star \star}$ & 55,0 & & GAB & Bile Allogho, 1999 \\
\hline Ozigo & 42705 & 0,347 n.s. & 86,0 & 1,29 & 29,8 & $-0,283$ n.s. & 0,009 n.s. & 3,3 & & GAB & Bile Allogho, 1999 \\
\hline Sapelli & 40415 & 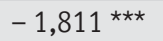 & 74,9 & 4,02 & 20,0 & 2,62 ** & $-0,046 * \star \star$ & 24,0 & & GAB & Bile Allogho, 1999 \\
\hline Ayous & 46106 & $-3,112^{\star \star \star}$ & 71,7 & 4,97 & 18,7 & $2,543^{*}$ & $-0,055^{\star \star \star}$ & 24,4 & & CAF & CTFT, 1964 \\
\hline Fraké & 39411 & $-1,088^{\star \star \star}$ & 91,8 & 1,81 & 15,3 & $-0,73$ n.s. & $-0,004$ n.s. & 0,6 & & CAF & CTFT, 1964 \\
\hline Iroko & 47031 & $-0,33$ n.s. & 88,7 & 2,94 & 12,6 & $-0,642$ n.s. & 0,003 n.s. & 0,1 & & CAF & CTFT, 1964 \\
\hline Sapelli & 39344 & $-0,345$ n.s. & 89,2 & 3,38 & 15,8 & $-1,632$ n.s. & 0,013 n.s. & 2,3 & & CAF & CTFT, 1964 \\
\hline Ayous & 39609 & 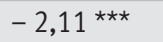 & 83,9 & 4,06 & 20,2 & 0,308 n.s. & $-0,024^{*}$ & 5,6 & & CIV & CTFT, 1967 \\
\hline Azobé & 41432 & $1,406^{\star \star}$ & 80,4 & 3,12 & 15,8 & $-4,503^{\star \star}$ & $0,054^{\star \star \star *}$ & 26,4 & & $\mathrm{CIV}$ & CTFT, 1967 \\
\hline Fraké & 46677 & 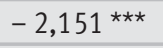 & 59,7 & 3,16 & 17,9 & $3,657^{\text {***}}$ & $-0,071$ *** & 59,7 & & CIV & CTFT, 1967 \\
\hline Iroko & 44483 & $-4,64^{\star \star \star}$ & 31,4 & 6,13 & 26,1 & $5,833^{* \star}$ & $-0,096^{\star \star \star}$ & 47,5 & & CIV & CTFT, 1967 \\
\hline Iroko & 48841 & $-4,436^{\star \star \star}$ & 46,4 & 5,71 & 28,1 & $3,653^{*}$ & $-0,074^{\star \star \star}$ & 35,3 & & CIV & CTFT, 1967 \\
\hline Sapelli & 46678 & 0,437 n.s. & 88,4 & 3,20 & 27,8 & 1,062 n.s. & $-0,006$ n.s. & 0,6 & & CIV & CTFT, 1967 \\
\hline Ayous & 42474 & $-2,307^{\star \star \star}$ & 81,9 & 4,23 & 17,0 & 0,844 n.s. & 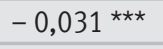 & 9,0 & & GNB & CTFT, 1986 \\
\hline Ayous & 42474 & $-2,307^{\star \star \star}$ & 81,9 & 4,23 & 17,0 & 0,844 n.s. & $-0,031$ *** & 9,0 & & CIV & CTFT, 1986 \\
\hline Fraké & 37160 & $-1,83^{\star \star \star}$ & 76,1 & 2,64 & 16,3 & $2,116^{*}$ & $-0,048^{* \star \star}$ & 41,1 & & GNB & CTFT, 1986 \\
\hline Iroko & 44261 & $-4,854^{\star \star \star}$ & 33,0 & 6,24 & 28,2 & $5,223^{* *}$ & $-0,092^{\star \star * \star}$ & 45,8 & & GNB & CTFT, 1986 \\
\hline Movingui & 46265 & $-2,278^{\star \star \star}$ & 17,6 & 3,76 & 23,6 & 1,121 n.s. & $-0,033$ n.s. & 4,2 & & GNB & CTFT, 1986 \\
\hline Movingui & 46265 & $-2,278^{\star \star \star}$ & 17,6 & 3,76 & 23,6 & 1,121 n.s. & $-0,033$ n.s. & 4,2 & & CIV & CTFT, 1986 \\
\hline Fraké & 43726 & $-1,524^{\star \star \star}$ & 83,8 & 2,27 & 15,0 & 1,276 n.s. & $-0,034 * \star \star$ & 26,4 & $\mathrm{x}$ & CIV & CTFT, 1988 \\
\hline Ayous & 43222 & $-3,045^{\star \star \star}$ & 69,8 & 5,00 & 18,5 & $3,202^{* *}$ & $-0,061 * \star \star *$ & 28,4 & & CIV & CTFT, 1990 \\
\hline Fraké & 44713 & $-4,235^{\star \star \star *}$ & 15,0 & 5,18 & 38,7 & 4,139 *** & $-0,103^{* \star *}$ & 74,8 & $x$ & GHA & Forster, 1994 \\
\hline Azobé & 40111 & $-3,622^{\star \star \star *}$ & 59,5 & 4,46 & 33,1 & $-5,403^{* *}$ & 0,016 n.s. & 2,7 & $x$ & GAB & Ngabou, 2011 \\
\hline Movingui & 41962 & $-2,207^{\star \star \star}$ & 18,1 & 3,72 & 23,4 & 1,171 n.s. & $-0,033$ n.s. & 4,1 & $x$ & GAB & Ngabou, 2011 \\
\hline Ozigo & 37014 & $-1,923 * \star \star \star$ & 61,7 & 2,28 & 68,7 & $-1,767^{\star \star}$ & $-0,002$ n.s. & 0,2 & $x$ & GAB & Ngabou, 2011 \\
\hline Sapelli & 43310 & $-2,836^{\star \star \star}$ & 68,6 & 4,62 & 21,3 & 1,871 * & $-0,049^{* * *}$ & 26,2 & $x$ & CAF & Ngabou, 2011 \\
\hline Taliivo & 49609 & $-0,307$ n.s. & 81,6 & 1,77 & 20,6 & 0,833 n.s. & $-0,011$ n.s. & 3,0 & $x$ & CAF & Ngabou, 2011 \\
\hline Taliivo & 49609 & $-0,307$ n.s. & 81,6 & 1,77 & 20,6 & 0,833 n.s. & $-0,011$ n.s. & 3,0 & $x$ & CAF & Ngabou, 2011 \\
\hline Assamela & 44446 & 0,129 n.s. & 73,1 & 2,15 & 22,9 & $-2,315^{\star}$ & 0,032 * & 11,7 & $x$ & CMR & ONADEF \\
\hline Iroko & 47449 & $-3,367^{\star \star \star *}$ & 63,0 & 4,71 & 21,2 & 2,777 n.s. & $-0,056$ *** & 24,1 & $x$ & CMR & ONADEF \\
\hline Movingui & 41233 & $-3,646^{\star \star \star}$ & $-24,9$ & 4,93 & 29,2 & 5,129 n.s. & $-0,086^{\star \star}$ & 22,6 & $x$ & CMR & ONADEF \\
\hline Ayous & 37172 & $-3,818^{\star \star \star}$ & 70,4 & 5,39 & 23,7 & 1,067 n.s. & $-0,048 * \star \star$ & 18,8 & & CMR & Palla, 2002a \\
\hline Ayous & 46713 & $-1,68 * \star \star$ & 85,0 & 3,89 & 17,4 & 0,857 n.s. & $-0,025^{*}$ & 6,0 & $x$ & CMR & API Dimako \\
\hline Ayous & 46711 & $-1,692^{\star \star \star *}$ & 84,9 & 3,90 & 17,4 & 0,866 n.s. & $-0,025^{\star}$ & 6,1 & & CMR & Palla, 2002a \\
\hline Ayous & 48646 & $-3,732$ *** & 60,7 & 5,62 & 20,4 & $3,744^{\star \star *}$ & $-0,073 * \star * \star$ & 36,3 & $x$ & CIV & Palla, 2002a \\
\hline Azobé & 37629 & $2,458^{* * *}$ & 73,9 & 4,20 & 20,4 & $-7,186^{\star \star \star \star}$ & $0,088^{\star \star \star *}$ & 47,1 & & CIV & Palla, 2002b \\
\hline Azobé & 38668 & 4,631 *** & 59,8 & 5,49 & 39,6 & $-3,106$ n.s. & 0,071 *** & 40,5 & & CMR & Palla, 2002b \\
\hline
\end{tabular}


Bois et Forêts des Tropiques - ISSN: L-0006-579X

Volume 338 - $4^{\text {rd }}$ quarter - December 2018 - p. 57-71

\begin{tabular}{|c|c|c|c|c|c|c|c|c|c|c|c|}
\hline $\begin{array}{l}\text { (Suite) } \\
\text { Essence } \\
\text { Sapelli }\end{array}$ & $\begin{array}{l}\text { Tarif ID } \\
37112\end{array}$ & $\begin{array}{l}\text { Biais } \\
0,016 \text { n.s. }\end{array}$ & $\begin{array}{l}\text { EFF } \\
88,9\end{array}$ & $\begin{array}{c}\text { RMSE } \\
3,16\end{array}$ & $\begin{array}{l}\text { ER\% } \\
20,1\end{array}$ & $\begin{array}{c}a \\
0,412 \text { n.s. }\end{array}$ & $\begin{array}{c}\mathbf{b} \\
-0,004 \text { n.s. }\end{array}$ & $\begin{array}{l}\mathbf{R}^{\mathbf{2}} \\
0,2\end{array}$ & Écorce & $\begin{array}{l}\text { Pays } \\
\text { CIV }\end{array}$ & $\begin{array}{l}\text { Référence } \\
\text { Palla, 2002c }\end{array}$ \\
\hline Sapelli & 37423 & 0,487 n.s. & 85,8 & 3,31 & 40,5 & $2,717^{\star \star \star}$ & $-0,023^{\star \star}$ & 7,3 & & CMR & Palla, 2002c \\
\hline Sapelli & 45947 & 0,281 n.s. & 89,9 & 3,23 & 15,9 & $-0,988$ n.s. & 0,013 n.s. & 2,4 & & $\mathrm{COG}$ & Palla, 2002c \\
\hline Sapelli & 46948 & $-3,185^{\star \star \star}$ & 65,0 & 4,90 & 23,1 & 1,851 * & $-0,052^{\star \star \star *}$ & 28,8 & & CMR & Palla, 2002c \\
\hline Sapelli & 42514 & 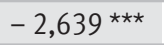 & 67,1 & 4,58 & 20,2 & 2,558 ** & $-0,054^{\star \star \star}$ & 30,3 & $x$ & CAF & Palla, 2002c \\
\hline Fraké & 37161 & $-3,828^{\star \star \star}$ & 38,7 & 4,54 & 38,0 & $2,486^{\star \star}$ & $-0,077^{\star \star \star}$ & 64,2 & & CIV & Pieper, 1992 \\
\hline Assamela & 40229 & $-1,248^{\star \star \star}$ & 45,9 & 2,32 & 25,6 & 0,249 n.s. & $-0,02$ n.s. & 5,3 & & GHA & Wong, 1990 \\
\hline Ayous & 47091 & $-1,316^{\star \star \star}$ & 88,1 & 3,76 & 15,9 & $-1,215$ n.s. & $-0,001$ n.s. & 0,0 & & GHA & Wong, 1991 \\
\hline Iroko & 40230 & 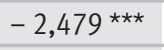 & 72,1 & 4,03 & 16,7 & $2,692^{*}$ & $-0,047^{\star \star \star *}$ & 18,4 & & GHA & Wong, 1992 \\
\hline Iroko & 40230 & 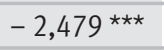 & 72,1 & 4,03 & 16,7 & 2,692 * & $-0,047^{\star \star \star}$ & 18,4 & & GHA & Wong, 1993 \\
\hline Sapelli & 40232 & $-0,026$ n.s. & 89,5 & 3,33 & 16,0 & $-1,388^{\star *}$ & $0,014^{\star \star}$ & 2,6 & & GHA & Wong, 1994 \\
\hline Sapelli & 40232 & $-0,026$ n.s. & 89,5 & 3,33 & 16,0 & $-1,388^{\star *}$ & 0,014 ** & 2,6 & & GHA & Wong, 1995 \\
\hline Sapelli & 40232 & $-0,026$ n.s. & 89,5 & 3,33 & 16,0 & $-1,388^{* \star}$ & 0,014 ** & 2,6 & & GHA & Wong, 1996 \\
\hline
\end{tabular}

Liste des références complètes pour les tarifs de cubage utilisés dans la plateforme GlobAllomeTree (Henry et al., 2013 ; http:// www.globallometree.org/D

API Dimako.

Bile Allogho J., 1999. Étude sur les ressources forestières du Gabon. Rome, Italie, FAO.

CTFT (Cirad), 1964. Inventaire forestier en Haute-Sangha, République Centrafricaine. Nogent-sur-Marne, France, Centre technique forestier tropical (Cirad).

CTFT (Cirad), 1967. Étude de reboisement et de protection des sols, République de Côte d'ivoire. Nogent-sur-Marne, France, Centre technique forestier tropical (Cirad).

CTFT (Cirad), 1986. Inventaire des ressources forestières de la région de la Lola, République de Guinée. Paris, France, Ministère de l'Agriculture, secrétariat d'État aux eaux et forêts, Centre technique forestier tropical (Cirad).

CTFT (Cirad), 1988. Croissance et productivité du Fraké en plantation. Centre technique forestier tropical de Côte d'Ivoire, Centre technique forestier tropical (Cirad).
CTFT (Cirad), 1990. Études sur la croissance du Samba (Tripolchiton scleroxylon) en plantation. Abidjan, Côte d'Ivoire, Centre technique forestier tropical (Cirad), Programme aménagement et gestion forestière.

Forster H., 1994. Teak volume tables for Odomi and Kabo river forest reserves. GTZ.

Ngabou B. P., 2011. Equation obtained from GTN. Origine et fiabilité des tarifs de cubage utilisés au Gabon pour les inventaires d'aménagement et d'exploitation. Rapport de stage, École nationale des eaux et des forêts.

ONADEF

Palla F., Louppe D., 2002a. Obéché. Cirad.

Palla F., Louppe D., Doumenge C., 2002b. Azobé. Cirad.

Palla F., Louppe D., Forni E., 2002c. Sapelli. Cirad.

Pieper Y., Laumans P., 1992. Contribution à l'élaboration d'un tarif de cubage multi-espèces pour les forêts classées de l'Est de la Côte d'Ivoire. Rapport IFCE n 3 , SODEFOR, GTZ, DFS.

Wong J. L. G., 1990. Forest resources management project temporary sample plot inventory computer program manual. ODA (UK).

\section{Annexe 3}

Matrice de variance-covariance des paramètres des tarifs de cubage ajustés. Les valeurs des paramètres ajustés $a, b$, $c, \delta$ et $\sigma$ sont indiquées ainsi que les variances (e.x. VAR ${ }_{a}$ et covaricances (e.x. VAR ${ }_{a b}$ ).

\begin{tabular}{|c|c|c|c|c|c|c|c|c|c|c|c|}
\hline Essence & $a$ & $V A R_{a}$ & $\boldsymbol{b}$ & $V A R_{b}$ & $c$ & $V A R_{c}$ & $V A R_{a b}$ & $V A R_{b c}$ & $V A R_{a c}$ & $\sigma$ & $\delta$ \\
\hline Assamela & & & $-6,56 e-03$ & $1,43 e-05$ & $1,14 \mathrm{e}-03$ & $5,37 e-09$ & & $-2,46 e-07$ & & $4,56 e-05$ & 1,191 \\
\hline Ayous & & & $-2,87 e-02$ & $8,96 e-06$ & $1,72 \mathrm{e}-03$ & 2,11e-09 & & $-1,22 \mathrm{e}-07$ & & $2,91 \mathrm{e}-05$ & 1,223 \\
\hline Azobé & & & $1,43 e-02$ & $8,11 \mathrm{e}-05$ & $9,35 \mathrm{e}-04$ & $9,46 e-09$ & & $-8,46 e-07$ & & $1,36 \mathrm{e}-05$ & 1,263 \\
\hline Fraké & & & $-1,32 \mathrm{e}-02$ & $4,20 e-05$ & $1,59 e-03$ & 8,88e-09 & & $-5,80 e-07$ & & $1,29 e-04$ & 1,040 \\
\hline Iroko & & & $-2,00 e-02$ & $4,97 e-05$ & $1,44 \mathrm{e}-03$ & $6,68 \mathrm{e}-09$ & & $-5,42 e-07$ & & $2,95 \mathrm{e}-05$ & 1,202 \\
\hline Movingui & & & 5,07e-03 & $8,43 e-04$ & 1,07e-03 & 7,87e-08 & & $-8,01 e-06$ & & $2,32 \mathrm{e}-02$ & 0,526 \\
\hline Okan & & & $-7,94 \mathrm{e}-03$ & $1,80 e-05$ & 1,19e-03 & $3,15 e-09$ & & $-2,12 e-07$ & & $6,99 e-05$ & 1,136 \\
\hline Ozigo & & & $-8,48 e-03$ & $2,86 e-05$ & $9,94 \mathrm{e}-04$ & 7,08e-09 & & $-4,15 e-07$ & & $8,47 e-04$ & 0,835 \\
\hline Padouk & & & $-1,37 e-03$ & $1,11 \mathrm{e}-04$ & $1,28 \mathrm{e}-03$ & $1,78 \mathrm{e}-08$ & & $-1,36 \mathrm{e}-06$ & & $6,38 \mathrm{e}-05$ & 1,137 \\
\hline Sapelli & $-2,79 e-01$ & $2,13 e-01$ & $-1,01 \mathrm{e}-02$ & $2,57 e-04$ & $1,36 \mathrm{e}-03$ & $1,43 e-08$ & $-7,26 \mathrm{e}-03$ & $-1,85 e-06$ & $5,05 e-05$ & $5,27 e-05$ & 1,164 \\
\hline Tali ivo & & & $-1,02 \mathrm{e}-02$ & $8,70 \mathrm{e}-05$ & 8,83e-04 & 8,71e-09 & & $-8,38 \mathrm{e}-07$ & & $5,76 \mathrm{e}-03$ & 0,614 \\
\hline Tali sua & & & $-2,35 e-02$ & $3,22 \mathrm{e}-05$ & $1,27 e-03$ & 5,63e-09 & & $-4,12 \mathrm{e}-07$ & & $1,27 \mathrm{e}-04$ & 1,041 \\
\hline
\end{tabular}




\section{Références bibliographiques}

Akindele S., LeMay V., 2006. Development of tree volume equations for common timber species in the tropical rain forest area of Nigeria. Forest Ecology and Management, 226: 41-48. https://doi.org/10.1016/j.foreco.2006.01.022

ATIBT, 2012. La lettre de l'ATIBT n 34. Nogent-sur-Marne, France, ATIBT.

De Wasseige C., De Marcken P., Bayol N., Hiol Hiol F., Mayaux P., Desclée B., et al., 2012. Les forêts du Bassin du Congo État des forêts 2010. OFAC, JRC-European Commission, 274 p. https://www.observatoire-comifac.net/docs/edf2010/FR/ Etat-des-forets 2010.pdf

De Wasseige C., Devers D., De Marcken P., Eba'a Atyi R., Nasi R., Mayaux P., 2009. Les forêts du Bassin du Congo - État des Forêts 2008. OFAC, JRC-European Commission, 426 p. https://www.observatoire-comifac.net/publications/ edf/2008

Durrieu de Madron L., Forni E., 1997. Aménagement forestier dans l'est du Cameroun. Bois et Forêts des Tropiques, 254 : 39-50. https://doi.org/10.19182/bft1997.254.a19897

FAO, 2003. Code régional d'exploitation forestière à faible impact dans les forêts denses tropicales humides d'Afrique centrale et de l'Ouest. Rome, Italie, FAO, CEE, 152 p.

Fargeot C., Forni E., Nasi R., 2004. Réflexions sur l'aménagement des forêts de production dans le bassin du Congo. Bois et Forêts des Tropiques, 281 : 19-34. https://doi. org/10.19182/bft2004.281.a20223

Fayolle A., Rondeux J., Doucet J.-L., Ernst G., Bouissou C., Quevauvillers S., et al., 2013. Réviser les tarifs de cubage pour mieux gérer les forêts du Cameroun. Bois et Forêts des Tropiques, 317 : 35-49. https://doi.org/10.19182/ bft2013.317.a20521

Henry M., Bombelli A., Trotta C., Alessandrini A., Birigazzi L., Sola G., et al., 2013. GlobAllomeTree: international platform for tree allometric equations to support volume, biomass and carbon assessment. iForest - Biogeosciences and Forestry, 6: 326-330. https://doi.org/10.3832/ifor0901-006

Henry M., Picard N., Trotta C., Manlay R., Valentini R., Bernoux M., et al., 2011. Estimating tree biomass of sub-Saharan African forests: a review of available allometric equations. Silva Fennica, 45: 477-569. https://www.silvafennica.fi/ pdf/article38.pdf

Karsenty A., Gourlet-Fleury S., 2006. Assessing sustainability of logging practices in the Congo Basin's managed forests: the issue of commercial species recovery. Ecology and Society, 11 (1): 26. http://www.ecologyandsociety.org/ vol11/iss1/art26/

Letouzey R., 1985. Notice de la carte phytogéographique du Cameroun au 1: 500 000. Toulouse, France, Institut de la carte internationale de la végétation.

Louppe D. (coord.), Mille G. (coord.), 2015. Mémento du forestier tropical. Versailles, France, Éditions Quæ, 1200 p.

Nasi R., Billand A., van Vliet N., 2012. Managing for timber and biodiversity in the Congo Basin. Forest Ecology and Management, 268: 103-111. https://doi.org/10.1016/i. foreco.2011.04.005

Palm R., 1982. Influence de la formule de cubage et de la longueur des billons sur la détermination du volume des arbres abattus. Annales des Sciences Forestières, INRA/EDP Sciences, 39 (3) : 231-238. https://hal.archives-ouvertes. $\mathrm{fr} /$ hal-00882278/document

Pérez M. R., de Blas D. E., Nasi R., Sayer J. A., Sassen M., Angoué C., et al., 2005. Logging in the Congo Basin: a multicountry characterization of timber companies. Forest Ecology and Management, 214: 221-236. https://doi. org/10.1016/i.foreco.2005.04.020

Picard N., Saint-André L., Henry M., 2012. Manuel de construction d'équations allométriques pour l'estimation du volume et la biomasse des arbres : de la mesure de terrain à la prédiction. Cirad, FAO, 210 p. http://www.fao.org/ docrep/018/i3058f/i3058f.pdf

Pinheiro J., Bates D., DebRoy S., Sarkar D., R Core Team, 2012. nlme: Linear and Nonlinear Mixed Effects Models. $\mathrm{R}$ package version 3.1-137. https://CRAN.R-project.org/ package $=$ nlme

R Core Team, 2016. R: A Language and Environment for Statistical Computing. Vienna, Austria, R Foundation for Statistical Computing.

Rondeux J., 1999. La mesure des arbres et des peuplements forestiers. Gembloux, Belgique, Les Presses agronomiques de Gembloux, 522 p.

Sist P., 2000. Les techniques d'exploitation à faible impact. Bois et Forêts des Tropiques, 265 : 31-43. https://doi. org/10.19182/bft2000.265.a20036

Tchatat M., Ndoumbe Nkeng M., Abena J. C., Foahom B., 2008. Volumes de bois autorisés à l'exploitation au Cameroun : détermination des valeurs maximales de dépassement tolérable. Bois et Forêts des Tropiques, 295 : 35-46. https://doi.org/10.19182/bft2008.295.a20390

Venables W. N., Ripley B. D., 2013. Modern applied statistics with S-PLUS. Springer Science \& Business Media, 450 p.

Zianis D., Muukkonen P., Mäkipää R., Mencuccini M., 2005. Biomass and stem volume equations for tree species in Europe. Silva Fennica, Monographs 4, 63 p. http://www. metla.fi/silvafennica/full/smf/smf004.pdf

Bois et Forêts des Tropiques - Revue scientifique du Cirad 